\title{
A Quantum Sensor for Neutrino Mass Measurements
}

\section{Juan Manuel Cornejo and Daniel Rodríguez}

Departamento de Física Atómica Molecular y Nuclear, Universidad de Granada, 18071 Granada, Spain

Correspondence should be addressed to Daniel Rodríguez, danielrodriguez@ugr.es

Received 6 July 2012; Accepted 7 September 2012

Academic Editor: Jose Bernabeu

Copyright (C 2012 J. M. Cornejo and D. Rodríguez. This is an open access article distributed under the Creative Commons Attribution License, which permits unrestricted use, distribution, and reproduction in any medium, provided the original work is properly cited.

There are few experiments aiming at determining directly the mass of the electron antineutrino with a sensitivity of $0.2 \mathrm{eV}$ by analyzing the end of the $\beta$-decay spectrum of specific nuclei. This sensitivity can be only reached if the uncertainties arising from systematic effects are very small and very well determined. The same holds for experiments aiming at improving the sensitivity in the determination of the mass of the electron neutrino using electron-capture $(\epsilon)$-decaying nuclei. One important input in these cases is an accurate $Q$-value of the decay which can be unambiguously determined from the difference of the mass of the mother and the daughter nuclei by means of Penning traps. In order to reach the required sensitivity, a novel device called Quantum Sensor is under construction at the University of Granada (Spain). The device will allow measuring atomic masses, and therefore $Q$-values from decays with unprecedented accuracy and sensitivity, using fluorescence photons from a laser-cooled ion instead of electronic detection. This paper will give an overview on $Q$-value measurements performed with Penning traps, relevant for neutrino mass spectrometry, describing the Quantum Sensor and the facility under construction. It will end by presenting the status of the project.

\section{Introduction}

Experimental results from atmospheric, solar, and reactor neutrino sources identified neutrino oscillations and established that neutrinos have rest masses [1-4]. However, neutrino oscillations are only sensitive to the differences of squared masses of different neutrino mass eigenstates. Absolute masses can be determined either by studying weak decays, that is, by determining the shape of the $\beta$-decay or, $X$-ray spectrum after electron capture ( $\epsilon$-decay), near the endpoint with good statistics and without systematic uncertainties and fit it with the neutrino mass as free parameter $[5,6]$, or being able to measure the half-live of nuclei undergoing double $\beta$-decay transformations $[7,8]$. The later is a second-order weak process which has a very small transition probability. While the fit of the $\beta$-decay spectrum, or X-ray spectrum from $\epsilon$-decay, allows for determining the mass 
independently whether the neutrino is a Dirac or Majorana particle, a successful half-life measurement from neutrinoless double $\beta$-decay $(0 v \beta \beta)$ or double electron-capture $(0 v \epsilon \epsilon)$ experiments would allow determining the Majorana neutrino mass, thus establishing the nature of the neutrino.

Either for single or double $\beta(\epsilon)$ decay transformations, a precise and unambiguous measurement of the $Q$-value of the transition, is of significant importance and can be provided only using Penning traps. These devices can provide the highest accuracy in the determination of the mass of atomic nuclei $[9,10]$ and, since the $Q$-value from $\beta$, double $\beta$, $\epsilon$, or double $\epsilon$ decay can be determined from the difference of the masses of the mother and the daughter nuclei, Penning traps provide the highest accuracy in the determination of this energy. The role of the $Q$-value in neutrino physics varies depending on the experimental goal. The use of the $Q$-value obtained from Penning-trap measurements to fit accurately the energy window to search events from $(0 v \beta \beta)$-decay on any of the eleven identified nuclides [11], or to conclude whether the predicted resonance enhancement for $(0 v \epsilon \epsilon)$-decay for some specific nuclides exists [7], requires a relative mass uncertainty $(\delta \mathrm{m} / \mathrm{m})$ ranging from $10^{-8}$ to $10^{-9}$, which is in reach with several Penning-trap facilities (see, e.g., $[12,13]$ ). For the determination of the mass of the electron (anti)neutrino from $\beta$ or $\epsilon$-decay, the candidates more suitable are those with small $Q$-values. Since a large number of possible sources of systematic errors exist, any additional information or constraint is highly wanted. For the determination of $m_{\bar{v}_{e}}$ from $\beta$-decay, a very important constraint is the endpoint energy $E_{0}\left(m_{\bar{v}_{e}}=0\right)$ for vanishing antineutrino mass as obtained by a measurement of the $Q$-value using high-accuracy mass spectrometry. The difference $E=E_{0}\left(m_{\bar{v}_{e}}=0\right)-E_{0}\left(m_{\bar{v}_{e}} \neq 0\right)$ must equal the mass of the electron antineutrino $m_{\bar{v}_{e}}$. From $\epsilon$-decay, $m_{v_{e}}$ can be obtained from the difference between the $Q$-value and the endpoint energy of the X-ray spectrum that originated from the decay of the daughter nucleus to the ground state. Either for $\beta$ or $\epsilon$ decay, the $Q$-value must be very accurately measured requesting in some cases relative mass uncertainties better than $10^{-11}$.

There are two experiments in the world under construction by large international collaborations which envisage to measure the mass of the electron antineutrino with a sensitivity of $0.2 \mathrm{eV}$ from single $\beta$-decay: the KArlsruhe TRItium Neutrino experiment (KATRIN) [14] and the Micro-calorimeter Array for a Rhenium Experiment (MARE) [15]. This sensitivity is one order of magnitude better compared to the present limit $\left(m_{\bar{v}_{e}}<2 \mathrm{eV}\right.$ at $95 \%$ C. L.) [5], achieved by use of electrostatic retardation spectrometers at Troitsk [16] and Mainz [17], measuring the end of the $\beta$-decay spectrum of tritium $\left(T_{1 / 2}=12.3\right.$ years $)$

$$
\mathrm{T}_{2} \longrightarrow\left({ }^{3} \mathrm{HeT}\right)^{+}+e^{-}+\bar{v}_{e}+18.5898(12) \mathrm{keV}
$$

(see [18]). The KATRIN collaboration will also measure the $\beta$-decay spectrum of tritium, but with an spectrometer scaled up in diameter by a factor of 10 compared to the one in Mainz. The main spectrometer vessel has a diameter of $10 \mathrm{~m}$ and a length of $24 \mathrm{~m}$, making it the largest mass spectrometer in the world. The MARE collaboration uses a totally different approach based on bolometers to measure the end of the $\beta$-decay spectrum of rhenium $\left(T_{1 / 2}=\right.$ $43.2 \times 10^{9}$ years)

$$
{ }^{187} \mathrm{Re} \longrightarrow{ }^{187} \mathrm{Os}+e^{-}+\bar{\nu}_{e}+2.4653(17) \mathrm{keV}
$$


(see [19]). There are two experiments from the same collaboration preceding MARE, called MANU [20] and MIBETA [19]. Measurements with MANU have yielded an upper limit for $m_{\bar{v}_{e}}$ of $26 \mathrm{eV}$ using a crystal of $1.5 \mathrm{mg}$ of metallic rhenium cooled down to $60 \mathrm{mK}$ with an activity of $1 \mathrm{~Bq}$. The results with the MIBETA detector constrained further the upper limit down to $15 \mathrm{eV}$. MIBETA is comprised of $10 \mathrm{AgReO}_{4}$ crystals, $300 \mu \mathrm{g}$ each. The MARE collaboration has also as final goal the measurement of the mass of the electron antineutrino with a sensitivity of $0.2 \mathrm{eV}[15,21]$, but it will develop in two steps, that is, MARE I aiming at reaching $2 \mathrm{eV}$ and MARE II aiming at improving this sensitivity by a factor of 10 .

The MARE collaboration also plans to utilize the same technique, based on bolometers, to determine the mass of the electron neutrino with high sensitivity by measuring the $\epsilon$-decay from ${ }^{163} \mathrm{Ho}\left(T_{1 / 2}=4.5 \times 10^{3}\right.$ years $)$

$$
{ }^{163} \mathrm{Ho}+e^{-} \longrightarrow{ }^{163} \mathrm{Dy}+v_{e}+2.80(5) \mathrm{keV}
$$

(see [6]). Though the $Q$-value is close to the one from the decay of ${ }^{187} \mathrm{Re}$, the half-life is seven orders of magnitude shorter. The main difficulty seems to be the implantation of pure ${ }^{163} \mathrm{Ho}$ on the detector, as this radioisotope is produced in reactions with strong isobaric contamination [6]. Very recently, a group at the Kirchhoff-Institute for Physics in Heidelberg (Germany) has presented the use of low-temperature metallic magnetic calorimeters to measure the spectra following the $\epsilon$-decay of ${ }^{163} \mathrm{Ho}$ (as well as the $\beta$-decay of ${ }^{187} \mathrm{Re}$ ) [22]. The group succeeded to implant the ${ }^{163} \mathrm{Ho}$ into the absorber of the detector. The experiment yielded a $Q$-value of $2.80(8) \mathrm{keV}$. Though some technical problems arising from the isobaric contamination of the sample have to be solved, the results are already very promising. The group at the Kirchhoff-Institute for Physics together with other groups has gathered in the $\mathrm{ECHO}$ (Electron Capture HOlmium experiment) Collaboration aiming at reaching in a short time scale a sensitivity on $m_{v_{e}}$ of a few $\mathrm{eV}$.

The $Q$-value given in (1.1) with $\delta Q=1.2 \mathrm{eV}$ has been determined from the difference of the masses of ${ }^{3} \mathrm{H}\left(\delta m / m=8.3 \times 10^{-10}\right)$ and ${ }^{3} \mathrm{He}\left(\delta m / m=8.6 \times 10^{-10}\right)$ using the SMILETRAP facility $[18,23]$. A dedicated Penning-trap experiment is in operation at the Max-Planck Institute for Nuclear Physics (MPIK) in Heidelberg in the group of K. Blaum in order to reach an uncertainty in the determination of the $Q$-value of $0.1 \mathrm{eV}$ [24], which corresponds to a relative mass uncertainty of a few times $10^{-11}$. Besides the group at the MPIK, the group of E.G. Myers at Florida State University is also preparing an experiment to measure the $Q$-value of the tritium decay using a Penning trap [25].

The $Q$-value given in (1.2) with $\delta Q=1.7 \mathrm{eV}$ has been obtained from the same experiment as the upper limit for the mass of the electron antineutrino [19]. Similarly, the $Q$-value given in (1.3) with $\delta Q=50 \mathrm{eV}$ has been obtained from the measurements performed with a bolometer setup [26]. In both cases, a different approach should be used, in order to obtain a result independent of systematic sources arising from the detectors. However, the masses of the nuclei involved in the rhenium and holmium decay are a factor of 60 and 54, respectively, larger compared to the masses of ${ }^{3} \mathrm{H}$ and ${ }^{3} \mathrm{He}$. In the case of ${ }^{187} \mathrm{Re}$, this forces the determination of the mass with $\delta \mathrm{m} / \mathrm{m} \sim 1.0 \times 10^{-11}$ to use the $Q$-value in combination with the outcomes from the MARE I campaign, and $\delta m / m \sim 1.0 \times 10^{-12}$ in order to complement the results obtained in the second campaign (MARE II). A relative mass uncertainty of $10^{-11}$ is within the present limit of Penning trap mass spectrometry and only demonstrated for low or medium mass-to-charge ratios [10]. A relative mass uncertainty of $1.0 \times 10^{-12}$ is not in reach. Note that almost the same applies for the decay of ${ }^{163} \mathrm{Ho}$. 
With the aim to improve the current performance, a novel device called Quantum Sensor is under development at the University of Granada using laser cooling in combination with the Penning trap [27]. The measurement of the atomic mass will be carried out using a system comprising two Penning traps with a common endcap, one for the ion under investigation and the other to confine $\mathrm{a}^{40} \mathrm{Ca}^{+}$ion which is laser-cooled to $\mathrm{mK}$ temperatures and will act as sensor. Since the ions are coupled via their axial oscillation frequencies, it is possible to exchange their motional energies. The cyclotron motion of the ion under investigation is transformed into axial motion along the magnetic field lines of the Penning trap and coupled to the sensor ion by the image current induced in the common endcap. The axial motion of the sensor ion in turn is monitored spatially resolved by its fluorescence light. In this way the detection of phonons is upgraded to a detection of photons.

\section{The Penning Trap and the Quantum Sensor}

In a Penning trap, charged particles are stored by the combination of a strong homogeneous magnetic field $(\vec{B})$ and a nearly perfect quadrupole electrostatic field $[28,29]$. The magnetic field is generated by a superconducting solenoid while the quadrupole field is performed by applying DC potentials to a set of electrodes with hyperbolical shapes as shown in the left side of Figure 1. In order to confine particles with positive polarity, the DC potential is applied either to the endcaps with respect to the ring electrode at ground (positive polarity) or to the ring with respect to the endcaps at ground (negative polarity). The right side of Figure 1 shows the electronic representation of a single ion with mass $m$ and electronic charge $q$ oscillating in the trap with a frequency $\omega$ [30].

The motion of any ion with mass-to-charge ratio $m / q$ stored in an ideal Penning trap can be described as the superposition of three independent motions, one in the direction of the magnetic field lines, called axial motion, with a characteristic oscillation frequency

$$
v_{z}=\frac{1}{2 \pi} \sqrt{\frac{q U}{m d^{2}}},
$$

where $d$ is a parameter related to the characteristics dimensions of the trap by

$$
d=\sqrt{\frac{z_{0}^{2}}{2}+\frac{r_{0}^{2}}{4}},
$$

and two motions in the radial plane, which are named modified cyclotron and magnetron. Their characteristic frequencies are

$$
v_{ \pm}=\frac{v_{c}}{2} \pm \sqrt{\frac{v_{c}^{2}}{4}-\frac{v_{z}^{2}}{2}}
$$

where

$$
v_{c}=\frac{1}{2 \pi} \cdot \frac{q}{m} \cdot B
$$




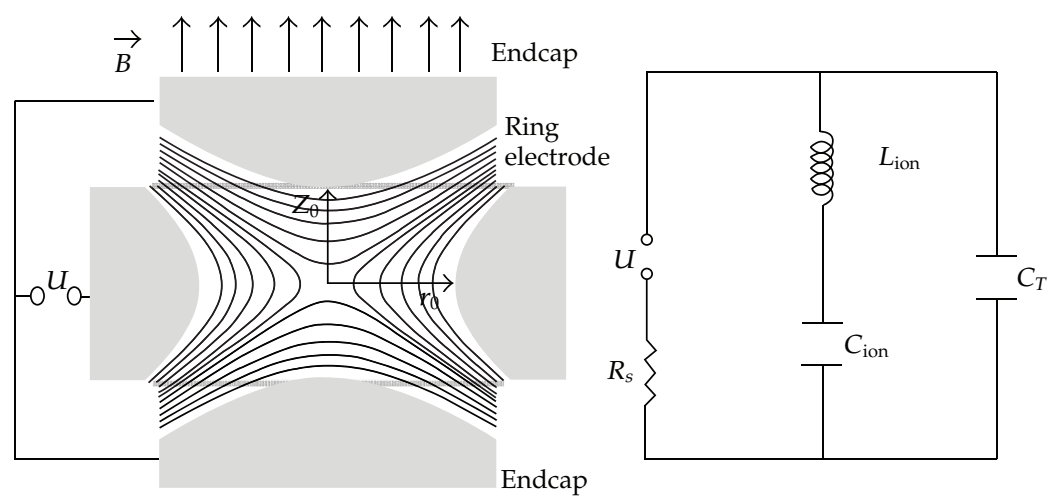

Figure 1: (right) Longitudinal projection of a hyperbolical Penning trap with cylindrical symmetry along the $z$-axis. $U$ is the DC potential applied to the endcaps with respect to the ring. Due to the shape of the electrodes, the equipotential lines are hyperboloids of revolution. $r_{0}$ and $z_{0}$ give the characteristics dimensions of the Penning trap and are related by $r_{0}=\sqrt{2} z_{0}$. (left) Equivalent circuit of the Penning trap with one stored ion. The ion can be described as an inductance $\left(L_{\text {ion }}=m\left(2 z_{0}\right)^{2} / q^{2}\right)$ in series with a capacitor $\left(C_{\text {ion }}=1 /\left(\omega^{2} L_{\text {ion }}\right)\right)$. $C_{T}$ gathers all the capacitances between the trap electrodes or between the electrodes and ground. $R_{S}$ is the internal resistance of the power supply.

is the cyclotron frequency of the ion moving in a magnetic field in the absence of any electric field.

Each of the motions has associated an oscillation amplitude $\left(\rho_{+}, \rho_{z}\right.$, and $\rho_{-}$, resp.). The reduction of these amplitudes, normally referred to as cooling, is important, especially to perform precision experiments, since when the ions move close to the center of the trap, they are less sensitive to inhomogeneities of the magnetic field or to imperfections of the quadrupole potential created by unavoidable deviations of the trap electrodes from the ideal hyperboloidal geometry. Any cooling mechanism should provide a nonconservative force acting in the direction of the motions so as to reduce the ion energy. Cooling to very low temperatures improves also the sensitivity of the detection by reducing the thermal-noise level, which is important for some detection techniques in Penning-trap mass spectrometry. There are several mechanisms to cool the ions in a trap.

(i) Cooling by collisions with buffer gas atoms or molecules [31] which is very general and applied in all Penning trap mass spectrometers installed at radioactive ion beam facilities.

(ii) Resistive cooling by connecting the endcaps of the Penning trap, which has a capacitance $C$, with an outer inductance $L$, such that the oscillation frequency of the ion equals that of the LC-circuit [30].

(iii) Laser cooling, provided the ionic level scheme of the ion allows for optical excitation [32]. This cooling method can provide the by far lowest temperatures.

(iv) Sympathetic cooling. Here the cooling of the ion of interest is due to Coulomb interaction with an ion which can be cooled directly. For example, in [33], $\mathrm{Hg}^{+}$ions were cooled by Coulomb interaction with laser-cooled $\mathrm{Be}^{+}$ions.

(v) Evaporative cooling. This cooling technique starts off with a cloud of ions. The hottest ions are forced to leave the trap until a single ion is left over which is the coldest one. 
In a Penning trap, after cooling, the mass-to-charge ratio of the ion of interest is determined from its cyclotron frequency provided the magnetic field strength is known. The magnetic field strength $B$ can be obtained from the cyclotron frequency measurement of an ion with well-known mass stored in the trap. The mass of the ion of interest $(m / q)^{\text {ion }}$ can be written as

$$
\left(\frac{m}{q}\right)^{\text {ion }}=\left(\frac{m}{q}\right)^{\mathrm{ref}} \cdot \frac{v_{c}^{\mathrm{ref}}}{v_{c}^{\text {ion }}}
$$

where the superscript ref represents the reference ion. The atomic mass can then be obtained by

$$
m_{\text {atomic }}=\frac{\nu_{c}^{\text {ref }}}{v_{c}^{\text {ion }}} \cdot\left(m_{\mathrm{ref}}-m_{e}-B_{e}\right)+m_{e}
$$

where $B_{e}$ is the binding energy of the outer electron which is very accurately known and $m_{e}$ is the mass of the electron measured with an uncertainty of about $10 \mathrm{meV}$. The cyclotron frequency can be measured using either the relationship

$$
v_{c}=v_{+}+v_{-}
$$

which only holds in the case of an ideal Penning trap [34], or the relationship

$$
v_{c}^{2}=v_{+}^{2}+v_{z}^{2}+v_{-}^{2}
$$

which is known as the invariance theorem and holds for the real Penning trap [28, 34]. Currently, there are two techniques which are used for the determination of $v_{c}$, namely, the time-of-flight (tof) resonance technique [35] used at the Penning-trap systems coupled to Radioactive Ion Beams (RIB) [29, 36] and the Induced-Image-Current (IIC) resonance technique applied to atomic ions from stable isotopes, molecular ions, or subatomic particles and antiparticles $[9,10,37,38]$. A third technique referred to as Quantum Sensor (QS) is under development to push the present performance in Penning-trap mass spectrometry [27].

\subsection{The TOF Resonance Technique}

It is based on the coupling of the modified-cyclotron and magnetron motions by applying an external radiofrequency (RF) quadrupole field [35]. After probing the ions with the RF field, the cyclotron frequency is obtained from the time-of-flight spectra of the ions ejected from the trap and registered with a microchannel plate detector located outside the magnetic field lines. The time-of-flight spectrum is measured for different $\mathrm{RF}$ frequencies $\left(v_{\mathrm{RF}}\right)$ around $v_{c}$. The mean time-of-flight of the distribution is plot versus $v_{\mathrm{RF}}$ and will be minimum for $v_{\mathrm{RF}}=v_{c}$ (resonance). There are variants of this technique which allows reaching a better relative mass uncertainty: the use of spatially separated quadrupole fields [39] for probing the ions or the use of higher-order multipolar fields $[40,41]$. The determination of the cyclotron frequency using the tof technique requires several tens of ions, and, with sufficient statistics, it is possible 


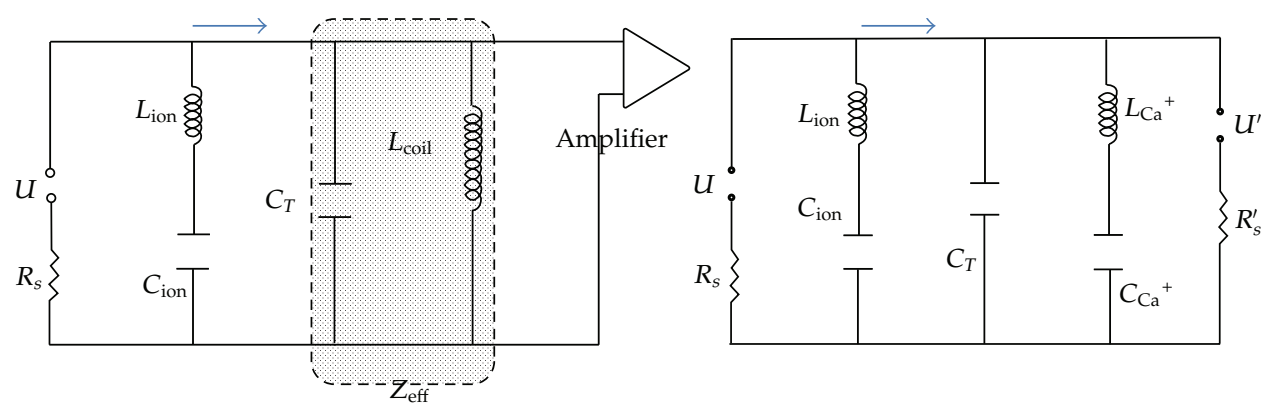

Figure 2: (right) Electronic equivalent of the ion in the trap attached to an external coil to apply the IIC technique. The inductance of the coil is taken so that the axial oscillation frequency of the ions in the trap is equal to the resonance frequency of the $L_{\text {coil }} C_{T}$-circuit. In this way, the circuit provides an effective impedance for the induced current (arrow in the top) before further amplification. The system must be operated at cryogenic temperature so that the electronic noise is minimum. (left) Electronic representation of the two ions connected via a common endcap. The energy is transferred from the ion of interest to the sensor ion within a time according to (2.9). The laser-cooled ion is at $\mathrm{mK}$ temperature and therefore more sensitive to the motion of the ion of interest. Since this is oscillating at very low energy (equivalent to $\mathrm{mK}$ temperature), any small transfer of energy will be already observed.

to attain a relative mass uncertainty $(\delta \mathrm{m} / \mathrm{m})$ in the order of $10^{-9}$, provided a careful study of the different sources of systematic uncertainties has been performed.

\subsection{The IIC Technique}

It is based on the resonant amplification of the current a charged particle induces on the endcap electrodes while it is oscillating in the trap at cryogenic temperature [42]. In order to detect such a minute current, an effective impedance is created with an external LC-circuit (see, e.g., [43]) or a tuned Superconducting Quantum Interference Device (SQUID) [42], in resonance with the axial oscillation of the ion, such that $v_{z}=1 /(2 \pi \sqrt{L C})$. Figure 2 shows, in the left side, the electronic representation. The axial oscillation frequency will be unfolded by applying a Fourier transformation to the current induced by the ion after flowing through the effective impedance $\left(Z_{\text {eff }}\right)$. Since $v_{c}$ is determined from $(2.8)$, one has to measure $v_{+}, v_{z}$, and $\nu_{-}$only by observing the axial frequency of the ion in resonance with the $L C$-circuit. This is done either by coupling the motions with an external pulse at $v_{z} \pm v_{+}$or $v_{z} \pm v_{-}$, after probing the ion motion with an RF field around the corresponding radial frequency, transferring in this way the energy from one radial motion to the axial motion and looking for the time to damp the amplitude of the axial signal [44-46], or by looking to a shift in the axial frequency which depends on the modified-cyclotron orbit [10]. This technique allows one to reach relative mass uncertainties in the order of $10^{-11}$ either by measuring the ion of interest and the reference ion sequentially in an ultrahigh stable magnetic field [10] or by storing both simultaneously in the trap [9].

\subsection{The QS Technique}

It is a novel technique for cooling, detection, and mass spectrometry of a single ion using a sensor ion $\left({ }^{40} \mathrm{Ca}^{+}\right)$which is laser-cooled to $\mathrm{mK}$ temperatures [27]. It is based on the axial coupling of two ions in different traps connected by a common endcap [47]. The electronical 
representation is shown in the right side of Figure 2. The ion of interest is probed by applying an external RF field in a similar way as with the IIC technique. After conversion to axial motion by applying an external pulse, the ion of interest transfers its energy to the sensor ion by making equal their axial oscillation frequencies. After the motional amplitudes between the ion of interest and the sensor ion are exchanged, the laser is $\mathrm{ON}$ and the fluorescence of the sensor ion is monitored with a CCD camera. The electronic detection of image charges is replaced by laser spectroscopic detection [27]. The circuit sketched in the right side of Figure 2 indicates that the transfer of the energy between the ions can be described from the coupling of the two circuits within a time called $t_{\mathrm{ex}}$ and given by [47]

$$
t_{\mathrm{ex}}=2 \pi^{2} v_{z} C \sqrt{L_{\mathrm{ion}} L_{\mathrm{Ca}+}} .
$$

In order to maintain the coupling and to transfer the energy, the oscillation frequencies of the two ions must be equal within

$$
\Delta v_{z}=\frac{1}{2 t_{\mathrm{ex}}}
$$

The QS technique has the following advantages [27].

(1) It is universal, independent of charge, and mass opposite to the IIC technique, not yet demonstrated for ions with mass-to-charge ratios above the one of ${ }^{133} \mathrm{Cs}^{2+}$ [48].

(2) A high signal-to-noise ratio can be realized for very small oscillation amplitudes compared to the IIC technique.

(3) The ion under investigation and the sensor ion are well separated and can be individually manipulated.

(4) The coupling of the two ions is well under control and can be changed easily in strength.

(5) The ion under investigation and the sensor ion can be in different charge states. Both should have such a charge-over-mass ratio that the axial frequencies can be tuned equal or to harmonics.

(6) The nonlinear Coulomb interaction of two ions stored in the same trap is avoided.

(7) Fluctuations of the magnetic field of the superconductor are cancelled in first approximation by a measurement of the cyclotron frequency of the sensor ion.

Items 1, 2, and 6 are the current factors limiting the most precise measurement by Penning traps [9]. The following section will show the measurements carried out using Penning traps which are related to neutrino physics and will also briefly underline the advantages of the QS technique compared to the IIC technique for applications to the measurement of the mass of the electron antineutrino.

\section{The Role of Penning Traps in Neutrino Physics}

Penning traps are devices which have been proven to contribute to the determination of the mass of the electron (anti)neutrino or to determine its nature (whether it is a Dirac or a Majorana particle) envisaged by several international collaborations, by 
performing extremely high-sensitive double- $\beta$-decay experiments to search the signature for a neutrinoless process, by studying the end of the single $\beta$-decay spectrum from weak transitions following the idea by E. Fermi, or by measuring the X-ray spectrum following electron capture processes. While the signature to establish the nature of the neutrino has not been observed unambiguously yet [11], the study of the $\beta$-decay spectrum has already permitted to set an upper limit for the mass of the electron antineutrino [5]. A recent experiment following $\epsilon$-decay for the determination of the mass of the electron neutrino seems very promising [22]. The role of Penning traps lies on the accurate and unambiguous determination of the $Q$-value of any $\beta(\epsilon)$ transformation (single or double) from the difference of the masses of the mother and the daughter nuclei. This determination does not suffer from systematic sources associated to detectors like intrinsic resolution, pileup effects, electronic noise. In addition, only a few ions from the daughter and the mother nuclei are needed. Furthermore, in the case of a double $\beta$ transformation, the Penning-trap $Q$-value determination can be performed in an infinitely shorter time scale compared to the expected half-lives of the mother nuclei, and if new candidates arise from theoretical predictions, the feasibility for the double decay can be easily cross-checked.

\subsection{Neutrinoless Processes in $\beta$ Transformations}

All the experiments in the world searching for a neutrinoless process concentrate on double $\beta^{-}$decay $(0 v \beta \beta)$, a process predicted for the first time in 1939 [49] and described by

$$
(A, Z) \longrightarrow(A, Z+2)+2 e^{-}+Q_{\beta \beta}
$$

$Q_{\beta \beta}$ is calculated from the mass excess $D$, which is defined as

$$
D(A, Z)=M(A, Z) c^{2}-A \cdot u-(E-Z) m_{e} c^{2},
$$

using the relationship

$$
Q_{\beta \beta}=D(A, Z)-D(A, Z+2) .
$$

The parameter $u$ in (3.2) is the atomic mass unit in $\mathrm{keV}$, and $E$ is the number of atomic electrons which appears from the fact that the mass excess is defined for neutral atoms. The probability for this transition is predicted to be higher compared, for example, to that of a neutrinoless double electron capture $(0 v \epsilon \epsilon)$ process pointed out for the first time in 1955 [50] and described by

$$
(A, Z)+2 e^{-} \longrightarrow(A, Z-2)+Q_{\epsilon \epsilon}
$$

$Q_{e \epsilon}$ can be also calculated from the mass excess $D$ using the relationship:

$$
Q_{\epsilon \epsilon}=D(A, Z)-D(A, Z-2) .
$$

The measurement of the well-defined energy $Q_{\beta \beta}$ or $Q_{\epsilon e}$ would imply the neutrino is a Majorana particle [51]. Table 1 shows in the last column the $Q$-values obtained by means of 
Penning traps using different facilities worldwide, SMILETRAP in Sweden [23], JYFLTRAP in Finland [52], SHIPTRAP in Germany [53], ISOLTRAP at CERN [54], or the MIT trap now relocated at Florida State University (FSU) [55]. Save the MIT trap at FSU, where the IIC technique is used, all the other facilities employ a variant of the tof technique [39-41]. The relative mass uncertainties from these measurements are always better than $1 \times 10^{-8}$. The column $\delta \bar{r} / \bar{r}$ in the table shows the relative uncertainty of the ratio between the cyclotron frequencies of the mother and the daughter nuclei. The resulting uncertainty in the $Q$-value is plotted also in Figure 3 (filled squares). Besides fixing accurately the energy window for observation of the events from running $(0 v \beta \beta)$-decay experiments [55], the $Q$-value can be used to (re)evaluate very accurately the half-life of the parent nuclei as already addressed in [56]. For a $(0 v \beta \beta)$-decay, $T_{1 / 2}$ can be given by

$$
\left[T_{1 / 2}^{0 v}\right]^{-1}=G^{0 v}\left(Q_{\beta \beta}, Z\right) g_{A}^{4}\left(M^{0 v}\right)^{2} \frac{\left\langle m_{v}\right\rangle^{2}}{m_{e}^{2}}
$$

where $G^{0 v}$ is a precisely calculated phase-space factor which depends on the $Q$-value of the transition, $g_{A}$ is the axial-vector coupling constant, and $M^{0 v}$ is the nuclear matrix element, which depends on the nuclear structure of the particular isotopes $(A, Z),(A, Z+1)$, and $(A, Z+2)$ under study. Details about these calculations and the models employed can be found elsewhere $[8,57]$. The probabilities for the $(0 v \beta \beta)$-transitions are smaller compared to the cases where $2 v$ are emitted. The latter is then more sensitive to be observed with the same experimental arrangement. Though $(0 v \beta \beta)$-transitions are expected with higher probability compared to $(0 v \epsilon \epsilon)$-decays, a possible resonance enhancement of this process might occur if the initial and final states of the transition are degenerate in energy as proposed in [7]. The process can be written as

$$
(A, Z)+2 e^{-} \longrightarrow(A, Z-2)^{*}+Q \longrightarrow(A, Z-2)+\left(Q_{\epsilon \epsilon}-E\right)
$$

where $(A, Z-2)^{*}$ denotes the excited state of the daughter nucleus, $Q$ is the energy released in such a decay which must be very small for resonance, and $E$ is the decay energy from the excited state to the ground state of the daughter nucleus. The excited state has a width $\Gamma$. In this process, the half-life reads

$$
\left[T_{1 / 2}^{0 v}\right]^{-1}=\frac{(\Delta M)^{2}}{\left(Q_{\epsilon \epsilon}-E\right)^{2}+\Gamma^{2} / 4} \Gamma
$$

where $\Delta M$ is the atom-mixing amplitude which is a function of several parameters, and $Q$ is the mass difference between the ground-state atoms $M(Z, A)$ and $M(Z-2, A)$. Using this formalism, the atom mixing amplitude is proportional to the effective neutrino mass. The process is enhanced if the difference $\left(Q_{\epsilon \epsilon}-E\right)$ is closer to zero. Several candidates were presented in [7] and some of them have been recently studied with Penning traps yielding in most of the cases a value for the half-life of the expected decay. All the $Q_{\epsilon \epsilon}$-values from Penning-trap mass spectrometry are also listed in Table 1 . Table 2 shows other double decays and the expected half-lives according to [58]. The $Q$-values are obtained from the latest Atomic Mass Evaluation [59] and the uncertainties are also shown in Figure 3 (filled circles). 
Table 1: $Q$-values for double $\beta$ transformations determined from Penning-trap mass measurements on the mother and the daughter nuclei. In case where the measurement was performed at two different Penningtrap facilities, only the value with smaller relative mass uncertainty is shown.

\begin{tabular}{|c|c|c|c|c|c|}
\hline $\begin{array}{l}\text { Double } \\
\text { decay }\end{array}$ & $\begin{array}{c}\text { Decay } \\
\text { type }\end{array}$ & $\begin{array}{c}T_{1 / 2} \text { (double decay) } \\
\text { (years) }\end{array}$ & $\begin{array}{c}\text { N.A. (mother) } \\
(\%)\end{array}$ & $\delta \bar{r} / \bar{r}$ & $\begin{array}{c}Q \text {-value } \\
(\mathrm{keV})\end{array}$ \\
\hline${ }^{76} \mathrm{Ge}^{-76} \mathrm{Se}^{(\mathrm{a})}$ & $0 v \beta \beta$ & $>7.2 \times 10^{20}[11]$ & 7.73 & $7.8 \times 10^{-10^{* *}}$ & $2039.006(50)[60]$ \\
\hline${ }^{74} \mathrm{Se}^{-74} \mathrm{Ge}^{(\mathrm{b})}$ & $0 v \in \epsilon$ & $\sim 5.0 \times 10^{43}[61]^{*}$ & 0.89 & $7.4 \times 10^{-10}$ & 1209.169 (49) [61] \\
\hline${ }^{96} \mathrm{Ru}^{-9}{ }^{96} \mathrm{Mo}^{(\mathrm{c})}$ & $0 v \in \epsilon$ & - & 5.54 & $1.4 \times 10^{-9}$ & $2714.51(13)[62]$ \\
\hline${ }^{100} \mathrm{Mo}^{-100} \mathrm{Ru}^{(\mathrm{b})}$ & $0 v \beta \beta$ & $>2.7 \times 10^{22}[11]$ & 9.82 & $1.8 \times 10^{-9}$ & 3034.40 (17) [63] \\
\hline${ }^{110} \mathrm{Pd}-{ }^{110} \mathrm{Cd}^{(\mathrm{d})}$ & $0 v \beta \beta$ & $6.8 \times 10^{23}[56]$ & 11.72 & $6.38 \times 10^{-9}$ & 2017.85 (64) [56] \\
\hline${ }^{112} \mathrm{Sn}-{ }^{112} \mathrm{Cd}^{(\mathrm{b})}$ & $0 v \in \epsilon$ & $5.9 \times 10^{29}[12]^{*}$ & 0.97 & $1.5 \times 10^{-9}$ & $1919.82(16)[12]$ \\
\hline${ }^{116} \mathrm{Cd}-{ }^{116} \mathrm{Sn}{ }^{(b)}$ & $0 v \beta \beta$ & $>8 \times 10^{21}[11]$ & 7.49 & $2.0 \times 10^{-9}$ & $2813.50(13)[64]$ \\
\hline${ }^{130} \mathrm{Te}-{ }^{130} \times \mathrm{e}^{(\mathrm{e})}$ & $0 v \beta \beta$ & $>2.2 \times 10^{21}[11]$ & 34.08 & $9.8 \times 10^{-11}$ & 2527.518 (13) [65] \\
\hline${ }^{136} \mathrm{Xe}^{136} \mathrm{Ba}^{(e)}$ & $0 v \beta \beta$ & $>1.6 \times 10^{22}[11]$ & 8.8573 & $7.9 \times 10^{-11^{* *}}$ & 2457.83 (37) [55] \\
\hline${ }^{136} \mathrm{Ce}-{ }^{136} \mathrm{Ba}{ }^{(b)}$ & Ovee & $>3.2 \times 10^{32}[66]^{*}$ & 0.185 & $3.8 \times 10^{-9}$ & 2378.53 (27) [66] \\
\hline${ }^{150} \mathrm{Nd}-{ }^{150} \mathrm{Sm}^{(\mathrm{b})}$ & $0 v \beta \beta$ & $>1.5 \times 10^{21}[11]$ & 5.638 & $1.4 \times 10^{-9}$ & 3371.38 (20) [67] \\
\hline${ }^{152} \mathrm{Gd}-{ }^{152} \mathrm{Sm}^{(\mathrm{c})}$ & $0 v \in \epsilon$ & $\sim 1.0 \times 10^{26}[13]^{*}$ & 0.20 & $1.3 \times 10^{-9}$ & 55.70 (18) [13] \\
\hline${ }^{156} \mathrm{Dy}_{-}{ }^{156} \mathrm{Gd}^{(\mathrm{c})}$ & $0 v \in \epsilon$ & $6.0 \times 10^{28}[68]^{* \#}$ & 0.053 & $7.0 \times 10^{-10}$ & 2005.95 (10) [68] \\
\hline${ }^{162} \mathrm{Er}^{162} \mathrm{Dy}{ }^{(\mathrm{c})}$ & $0 v \in \epsilon$ & - & 0.139 & $2.0 \times 10^{-9}$ & $1846.95(30)[62]$ \\
\hline${ }^{164} \mathrm{Er}^{-164} \mathrm{Dy}^{(\mathrm{c})}$ & $0 v \in \epsilon$ & $\sim 1.0 \times 10^{30}[69]^{*}$ & 1.601 & $7.6 \times 10^{-10}$ & 25.07 (12) [69] \\
\hline${ }^{168} \mathrm{Yb}^{-168} \mathrm{Er}^{(\mathrm{c})}$ & $0 v \in \epsilon$ & - & 0.123 & $1.6 \times 10^{-9}$ & $1409.27(25)[62]$ \\
\hline${ }^{180} \mathrm{~W}-{ }^{180} \mathrm{Hf}^{(\mathrm{c})}$ & $0 v \in \epsilon$ & $\sim 5.0 \times 10^{27}[70]^{*}$ & 0.12 & $1.6 \times 10^{-9}$ & $143.20(27)[70]$ \\
\hline
\end{tabular}

The superscript besides the nuclides in the column "Double decay" serves to indicate the facility where the measurements were performed: ${ }^{(\mathrm{a})}$ SMILETRAP, ${ }^{(\mathrm{b})}$ JYFLTRAP, ${ }^{(\mathrm{c})}$ SHIPTRAP, ${ }^{(\mathrm{d})}$ ISOLTRAP, and ${ }^{(\mathrm{e})}$ MIT trap at FSU. The natural abundances of the daughter nuclei are in general higher compared to those of the mother. ${ }^{(*)}$ Assuming $m_{v_{e}}=1 \mathrm{eV}$. ${ }^{(\#)}$ Partial half-life. ${ }^{(*)}$ Individual ratios are given with respect to a molecular ion.

Table 2: Expected half-lives for double $\beta$-transformations from [58]. The $Q$-values listed in the last column have been obtained using either (3.3) or (3.5) with the mass excess values and uncertainties quoted in the latest published volume of the Atomic Mass Evaluation [59]. One can observe that the uncertainties in the $Q$-values are one order of magnitude larger compared to those listed in Table 1.

\begin{tabular}{|c|c|c|c|c|c|}
\hline $\begin{array}{l}\text { Double } \\
\text { Decay }\end{array}$ & $\begin{array}{c}\text { Decay } \\
\text { Type }\end{array}$ & $\begin{array}{c}T_{1 / 2} \text { (double decay) } \\
\text { (years) }\end{array}$ & $\begin{array}{c}\text { N.A. (mother) } \\
(\%)\end{array}$ & $\delta M_{\text {daughter }} / M_{\text {daughter }}$ & $\begin{array}{c}Q \text {-value } \\
(\mathrm{keV})\end{array}$ \\
\hline${ }^{48} \mathrm{Ca}-{ }^{48} \mathrm{Ti}$ & $0 v \beta \beta$ & $>5.8 \times 10^{22}[11]$ & 0.187 & $8.3 \times 10^{-8}$ & $4273.7(4.1)$ \\
\hline${ }^{78} \mathrm{Kr}-{ }^{78} \mathrm{Se}$ & $0 v \in \epsilon$ & $>1.5 \times 10^{21}[11]$ & 0.375 & $1.5 \times 10^{-8}$ & $2846.4(2.0)$ \\
\hline${ }^{82} \mathrm{Se}-{ }^{82} \mathrm{Kr}$ & $0 v \beta \beta$ & $>3.6 \times 10^{23}[11]$ & 8.73 & $2.7 \times 10^{-8}$ & $2995.5(2.7)$ \\
\hline${ }^{96} \mathrm{Zr}^{-}{ }^{96} \mathrm{Mo}$ & $0 v \beta \beta$ & $>9.2 \times 10^{21}[11]$ & 2.80 & $2.6 \times 10^{-7}$ & $3347.7(3.4)$ \\
\hline${ }^{102} \mathrm{Pd}-{ }^{102} \mathrm{Ru}$ & $0 v \in \epsilon$ & - & 1.02 & $2.9 \times 10^{-8}$ & $1172.9(3.2)$ \\
\hline${ }^{106} \mathrm{Cd}-{ }^{106} \mathrm{Pd}$ & $0 v \in \epsilon$ & $>0.9 \times 10^{19}[58]$ & 1.25 & $5.7 \times 10^{-8}$ & $2770(7)$ \\
\hline${ }^{128} \mathrm{Te}^{128} \mathrm{Xe}$ & $0 v \beta \beta$ & $>1.6 \times 10^{24}[11]$ & 31.74 & $1.5 \times 10^{-8}$ & $867.9(2.2)$ \\
\hline${ }^{130} \mathrm{Ba}-{ }^{130} \mathrm{Xe}$ & $0 v \in \epsilon$ & $>4 \times 10^{21}[58]$ & 0.106 & $2.3 \times 10^{-8}$ & $2620.1(2.9)$ \\
\hline${ }^{132} \mathrm{Ba}-{ }^{132} \mathrm{Xe}$ & $0 v \in \epsilon$ & $>3 \times 10^{20}[58]$ & 0.101 & $8.3 \times 10^{-9}$ & $845.7(1.5)$ \\
\hline
\end{tabular}

\subsection{Measurement of the Mass of the Electron Antineutrino from $\beta$-Decay}

A single $\beta$-decay process is described by

$$
(A, Z) \longrightarrow(A, Z+1)+e+\bar{v}_{e}+Q_{\beta},
$$




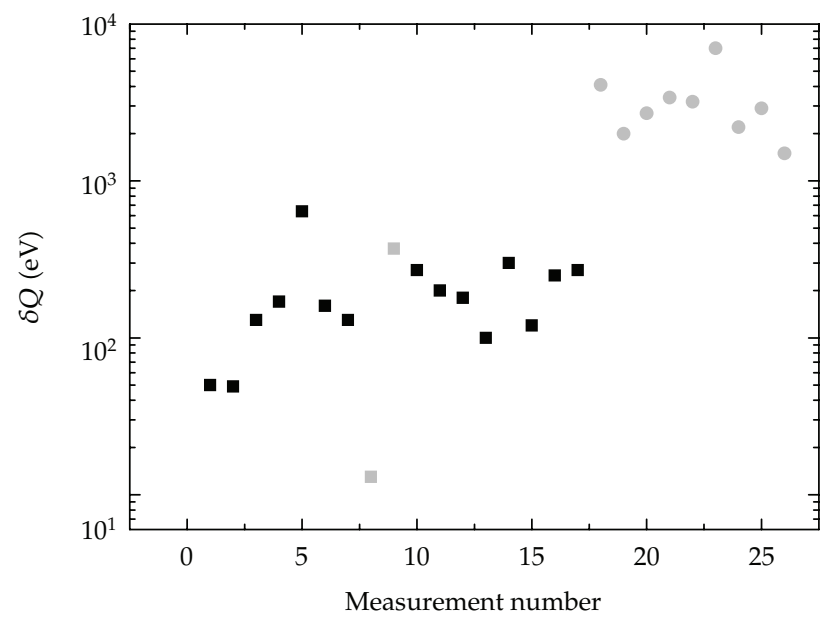

Figure 3: Uncertainty in the $Q$-values measured with Penning traps (filled squares) and from the latest published version of the Atomic Mass Evaluation (AME) [59] (filled circles). The squares in black and grey represent the results obtained using the tof and the IIC technique, respectively. The characteristics for the decays with the $Q$-values represented by filled circles are listed in Table 2.

where $Q_{\beta}$ is the energy released in the decay shared by the electron and the antineutrino, given by

$$
Q_{\beta}=\left[M(A, Z)-M(A, Z+1)-m_{e}-m_{\bar{v}_{e}}\right] c^{2},
$$

or, in terms of the mass excess, by

$$
Q_{\beta}=D(A, Z)-D(A, Z+1)-m_{\bar{v}_{e}} c^{2}
$$

We will follow the notation $Q_{\beta}\left(m_{\bar{v}_{e}}=0\right)=E_{0}$. In 1934 Enrico Fermi proposed the theory for $\beta$-decay. Within this theory and neglecting the energy of the recoil nucleus, the energy spectrum of electrons from the decay can be written as [15]

$$
N_{e}\left(Z, E_{e}, m_{v_{e}}\right)=p_{e} E_{e}\left(E_{0}-E_{e}\right) \sqrt{\left(E_{0}-E_{e}\right)^{2}-m_{v_{e}}^{2} c^{4}} F\left(Z, E_{e}\right) S\left(E_{e}\right)\left[1+\delta_{R}\left(Z, E_{e}\right)\right],
$$

where $p_{e}$ stands for the momentum of the electron, $F\left(Z, E_{e}\right)$ is called the Fermi function to account for the nuclear charge, $S\left(E_{e}\right)$ is the form factor of the $\beta$ spectrum, containing the nuclear matrix element, and $\delta_{R}\left(Z, E_{e}\right)$ is the radiative electromagnetic correction. One can study the spectrum using a transformation $K\left(E_{e}\right)$ such that

$$
K\left(E_{e}\right)=\left(E_{0}-E_{e}\right)\left(1-\frac{m_{v_{e}}^{2} c^{4}}{\left(E_{0}-E_{\beta}\right)^{2}}\right)^{1 / 4} .
$$

The representation of $K\left(E_{e}\right)$ versus $E_{e}$ is known as Kurie plot. By a detailed analysis of the shape of the end of the spectrum, the electron-neutrino mass can be unfolded. However, the 
information on the neutrino mass is only in the final part of the spectrum within an energy interval $\delta E$ containing a minute fraction of the total number of events

$$
F(\delta E) \approx 2\left(\frac{\delta E}{E_{0}}\right)^{3}
$$

For $\delta E=5 \mathrm{eV}$, the fraction of events is $3.9 \times 10^{-11}$ for KATRIN (1.1) and $1.6 \times 10^{-8}$ for MARE (1.2). This is very small and therefore requires a very careful measurement including an extensive study of the systematics effects, which in the case of MARE (the bolometer approach) are

(i) the response function of the detector,

(ii) the decay to excited states of the daughter nucleus,

(iii) the background due to cosmic rays and environmental radioactivity,

(iv) pile-up effects.

There is a paper devoted to KATRIN in this issue [71], and therefore only the experiment devoted to the $Q$-value measurement from mass difference of the pair ${ }^{3} \mathrm{H}-{ }^{3} \mathrm{He}$ using the UW-PTMS now in operation at the MPIK in Heidelberg in the division of Blaum [24] will be underlined to compare the method with the Quantum Sensor [27]. MARE, as KATRIN, aims at reaching finally a sensitivity of $0.2 \mathrm{eV} / \mathrm{c}^{2}$ in the measurement of the mass of the electron antineutrino. The first limit quoted by a group of the MARE collaboration for the mass of the electron antineutrino was $26 \mathrm{eV}$ using the MANU detector [20]. The energy resolution was varied between 30 and $50 \mathrm{eV}$ in the energy interval used for the fit, and the authors reported a $Q$-value of $2470 \pm 1$ (stat) \pm 4 (syst) $\mathrm{eV}$ where the systematic uncertainty was due to the influence of the nonlinearity of the energy together with the choice of the energy interval for the analysis. Furthermore, they stated a dependence of the endpoint energy on the energy resolution; a variation of $5 \mathrm{eV}$ in $\sigma$ resulted in a variation of $1 \mathrm{eV}$.

The lowest limit quoted up to date from the decay of ${ }^{187} \mathrm{Re}$, measured by another group of the MARE collaboration, is $15 \mathrm{eV}$ (90\% C. L.) using the MIBETA detector [19]. In this paper, the authors quoted a $Q$-value of $2465.3 \pm 0.5$ (stat) \pm 1.6 (syst) $\mathrm{eV}$, where the systematic uncertainty was determined from the uncertainties in the energy resolution $(28.5 \mathrm{eV}$ at $2.46 \mathrm{keV})$, in the response function of the detector, and in the shape of the background of the $\beta$-spectrum. Still, the limit for the mass of the electron antineutrino is above the aimed value. According to the MARE proposal [15], the final value will be reached in two steps: MARE I aiming at $m_{\bar{v}_{e}}<2 / c^{2}$ and MARE II aiming at $m_{\bar{v}_{e}}<0.2 / c^{2}$. In a very recent publication [72], a sensitivity of $4.5 \mathrm{eV}$ at $90 \% \mathrm{C}$. L. is claimed to be possible using eight arrays of detectors based on silicon-implanted thermistors in combination with $\mathrm{AgReO}_{4}$ crystals after three years of date taking. This is a continuation of the work carried out with the MIBETA detector (energy resolution below $30 \mathrm{eV}$ ). However, the goal to be pursued in the MARE II phase will need novel technology, based on the so-called kinetic inductance detectors. An arrangement of $10^{5}$ detectors $20 \mathrm{mg}$ each is needed according to [72].

Many factors and many preceding results have been taken into account in order to get an accurate limit for the mass of the electron antineutrino. However, a different approach to reach a $Q$-value $\left(E_{0}\right)$ with a better accuracy and more important not subject to detector resolution, background sources, or pile-up effect is highly desirable. For this purpose, there is currently one Penning trap in operation at the MPIK in Heidelberg to measure the mass 
difference of the pair ${ }^{3} \mathrm{H}-{ }^{3} \mathrm{He}$ for KATRIN [24]. The device has been proven to work at the University of Washington in Seattle, where the mass for ${ }^{4} \mathrm{He}^{2+}$ was measured with a relative mass uncertainty of $1.5 \times 10^{-11}$ [10]. This corresponds to an uncertainty in the mass excess $D$ of $0.06 \mathrm{eV}$, showing the feasibility to reach the accuracy required for KATRIN for the masses of ${ }^{3} \mathrm{H}$ and ${ }^{3} \mathrm{He}$ with the present performance of the spectrometer. In the case of MARE however, due to the higher masses of the daughter and the mother nuclei (a factor of 60 higher!), the uncertainty to be reached must be better and the new technique is needed. (In the case of the MARE project, the $Q$-value obtained from the mass difference as proposed in this paper, will differ slightly with the $Q$-value of the decay within the crystal. However, it can be corrected using an analytical expression given in the MARE proposal [15]. All the parameters involved are vey accurately known.)

The setup at the MPIK uses the IIC technique to measure the shift in the axial frequency from the following relationship:

$$
\frac{\delta v_{z}}{v_{z}}=\left[\frac{B_{2} d^{2}}{B_{0}}-3 C_{4}\left(\frac{v_{z}}{v_{+}}\right)^{2}\right] \frac{E_{+}}{q V_{0}},
$$

where the term $B_{2} d^{2} / B_{0}$ is smaller compared to $3 C_{4}\left(v_{z} / v_{+}\right)^{2}$, so that

$$
\frac{\delta v_{z}}{v_{z}} \approx 3 C_{4}\left(\frac{v_{z}}{v_{+}}\right)^{2} \frac{E_{+}}{q V_{0}}
$$

$C_{4}$ is a term defined elsewhere which can be below $10^{-4}$ in a so-called compensated Penning trap or in the order of $10^{-2}$ for an uncompensated one [73]. An external RF field serves to probe the modified-cyclotron motion. The radial energy $E_{+}$in (3.15) is maximum when $v_{\mathrm{RF}}=v_{+}$. Since the frequency $\boldsymbol{v}_{+}$is constant, an increase in energy is caused by the increase of the oscillation amplitude $\rho_{+}$. We use here (3.16) to illustrate the advantage of using the QS technique versus the ICC technique. Figure 4 shows the relative variation of the axial oscillation frequency versus the oscillation amplitude $\rho_{+}$for several values of $C_{4}$. The vertical dotted lines mark the requested ion's modified-cyclotron radius $\left(\rho_{+}\right)$in order to observe the induced current (IIC technique) [10] and the fluorescence (QS technique) [27]. As can be observed in the figure, the shift due to anharmonicities of the quadrupole field (following (3.16)) can be substantially reduced and one can aim at improving precision for the mass of the pairs ${ }^{187} \mathrm{Re}^{+}{ }^{187} \mathrm{Os}^{+}$. The QS technique, if successful, can be used therefore to contribute to the measurement pursued by the MARE collaboration, but can of course be applied to measure masses of $\epsilon$-decaying nuclei and contribute to the measurement of the mass of the electron neutrino.

\subsection{Measurement of the Mass of the Electron Neutrino from 6 -Decay}

A single electron capture process is given by

$$
(A, Z)+e^{-} \longrightarrow(A, Z-1)^{*}+Q_{\nu}
$$




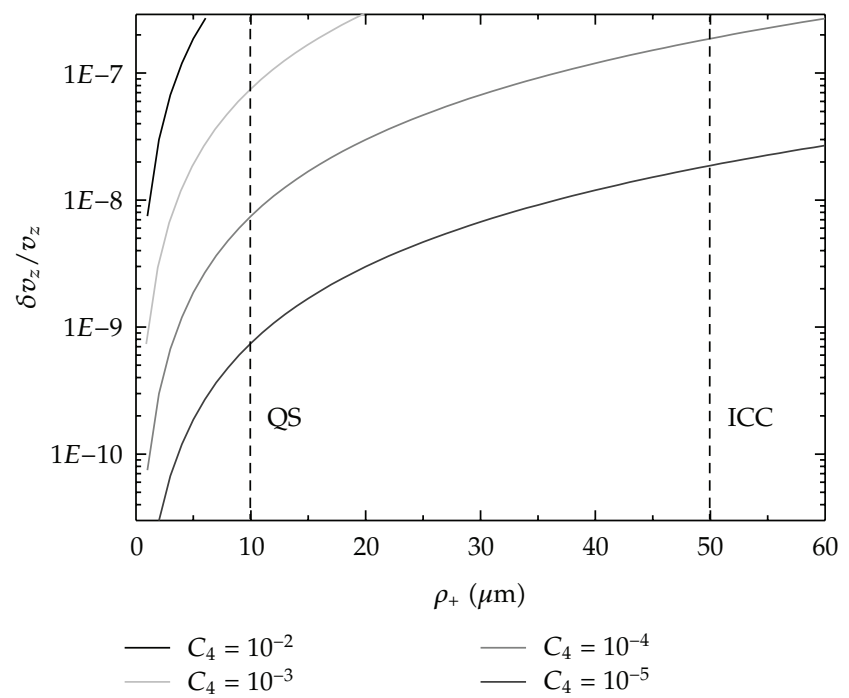

Figure 4: Relative variation of the axial frequency versus the modified-cyclotron radius of an ion with mass-to-charge ratio of 187 . The $y$-axis is plotted up to $\delta v_{z} / v_{z}=2.9 \times 10^{-7}$, which is the limit to maintain the coupling between the ion of interest and the sensor ion [27]. Using (2.9) and (2.10), the pairs ${ }^{187} \operatorname{Re}^{+}-$ ${ }^{40} \mathrm{Ca}^{+}$and ${ }^{187} \mathrm{Os}^{+}{ }^{40} \mathrm{Ca}^{+}$are coupled if their oscillation frequencies (we consider $v_{z}=100 \mathrm{kHz}$ ) are equal within $29 \mathrm{mHz}$ [27]. The ions are moving in a $7 \mathrm{~T}$ magnetic field, so that $v_{+}\left({ }^{187} \operatorname{Re}^{+}\right)=565282.43 \mathrm{~Hz}$.

where $Q_{v}$ comprises the energy and the rest mass of the emitted neutrino or by

$$
(A, Z)+e^{-} \longrightarrow(A, Z-1)+Q_{\epsilon}
$$

with

$$
Q_{\epsilon}=Q_{v}+B
$$

$B$ stands here for the total energy that originated from the decay of the excited state of the daughter nucleus. This energy is emitted in the form of X-rays and can be measured with calorimeters. Suitable candidates are those with very low $Q_{\epsilon}$-values and very small $Q_{v}$. As in $\beta$-decay, the end of the emission spectrum must be fitted in order to obtain the mass of the electron neutrino. For the analytical form see, for example, [6]. There are two collaborations aiming at measuring the mass of the electron neutrino with a sensitivity firstly of a few $\mathrm{eV}$ using the $\epsilon$-decay of ${ }^{163} \mathrm{Ho}$, MARE [6], and ECHO [22]. In the framework of the second collaboration, a Penning trap is under development in order to perform very accurate mass measurements on the parent and the daughter nuclei [74]. The discussion on accuracy is similar to the previous section.

Other candidates with low $Q_{e}$-values have been pointed out for the same kind of experiment: ${ }^{100} \mathrm{Pd}\left(T_{1 / 2}=3.63 \mathrm{~d}\right),{ }^{101} \mathrm{Rh}\left(T_{1 / 2}=3.3 \mathrm{y}\right),{ }^{131} \mathrm{Cs}\left(T_{1 / 2}=9,69 \mathrm{~d}\right),{ }^{134} \mathrm{Ce}\left(T_{1 / 2}=\right.$ $3.16 \mathrm{~d}),{ }^{57} \mathrm{~Tb}\left(T_{1 / 2}=71 \mathrm{y}\right),{ }^{159} \mathrm{Dy}\left(T_{1 / 2}=144,4 \mathrm{~d}\right),{ }^{161} \mathrm{Ho}\left(T_{1 / 2}=2,48 \mathrm{~h}\right),{ }^{163} \mathrm{Ho}\left(T_{1 / 2}=4570 \mathrm{y}\right)$, ${ }^{175} \mathrm{Hf}\left(T_{1 / 2}=70 \mathrm{~d}\right),{ }^{183} \operatorname{Re}\left(T_{1 / 2}=70 \mathrm{~d}\right),{ }^{194} \mathrm{Hg}\left(T_{1 / 2}=444 \mathrm{y}\right)$, or ${ }^{201} \mathrm{Tl}\left(T_{1 / 2}=3.04 \mathrm{~d}\right)$ [75]. Though they are not natural abundant isotopes, those with long half-life can still be practicable in the proposed system, provided we can get the source. 


\section{The Project TRAPSENSOR: Status}

The Quantum Sensor will be developed within the project TRAPSENSOR at the University of Granada (Spain). The project will be run in two steps. In the first one, two independent setups will be built:

(1) a commercial laser-desorption ion source, which is in operation at the University of Granada since July 2011, will be coupled to the Penning-trap beamline shown in Figure 6, which can allow for determination of $Q$-values using the tof technique to study, for instance, some of the cases listed in Table 2,

(2) a radiofrequency trap with a laser system to cool ${ }^{40} \mathrm{Ca}^{+}$to $\mathrm{mK}$ temperatures (Doppler cooling), comprising a set of diode lasers and associated equipment, which will serve to define the final geometry of the Quantum Sensor using the setup shown in Figure 7.

During the second stage of the project, the Quantum Sensor will substitute the precision Penning trap in Figure 6. The elements will be presented in the following.

\subsection{The Laser Desorption Ion Source}

The laser-desorption ion source is a commercial MALDI-tof system (model REFLEX III from Bruker). The acronym MALDI-tof stands for Matrix Assisted Laser Desorption Ionization Time Of Flight. In such device, a laser pulse on the sample produces the ions and serves as a trigger to measure the time the ions are registered in a microchannel plate detector at the end of a time-of-flight section. In July 2011 the production of $\mathrm{Re}^{+}$and $\mathrm{Os}^{+}$ions from naturally abundant isotopes at kinetic energies ranging from $20 \mathrm{keV}$ down to a few tens of $\mathrm{eV}$ was accomplished. The laser utilized in these experiments is a doubled-frequency Nd:YAG laser (model Brilliant from Quantel) with $\lambda=532 \mathrm{~nm}$, a pulse width of $\approx 4 \mathrm{~ns}$, and a repetition rate of $10 \mathrm{~Hz}$. Figure 5 shows the time-of-flight (tof) signal of $\mathrm{Re}^{+}$ions at $20 \mathrm{keV}$ (normal operation of the system), and $1 \mathrm{keV}$, which is the lower energy required to transport the ions through the transfer section and to inject them into the Penning traps (Figure 6). A rhenium foil was glued in the target plate of the MALDI apparatus and the laser energy was fixed to around $800 \mathrm{~mJ} / \mathrm{cm}^{2}$. Systematic investigations have been carried out varying the laser power, the ion energy, and the sample preparation, especially for osmium since it is supplied in small grains. The results together with the mechanical coupling to the Penning-trap beamline will be presented elsewhere [76].

\subsection{The Penning-Trap Beamline}

The layout of the Penning-trap beamline is shown in Figure 6. The system is comprised of a transfer section and two Penning traps housed in the same superconducting solenoid which has two homogeneous magnetic field regions separated by $20 \mathrm{~cm}$. The first region has a homogeneity of $1 \mathrm{ppm} / \mathrm{cm}^{3}$ while the second reaches $0.1 \mathrm{ppm} / \mathrm{cm}^{3}$. The superconducting magnet is similar to the one at SHIPTRAP [53], JYFLTRAP [52], and TRIGATRAP [77] and will be available at the University of Granada from October 2012. The magnetic field strength is $7 \mathrm{~T}$ in the region where the traps are located.

The first Penning trap in the beamline is made of a stack of cylinders in a similar way to the one conceptually designed for the preparation of the ions at MATS (precise 


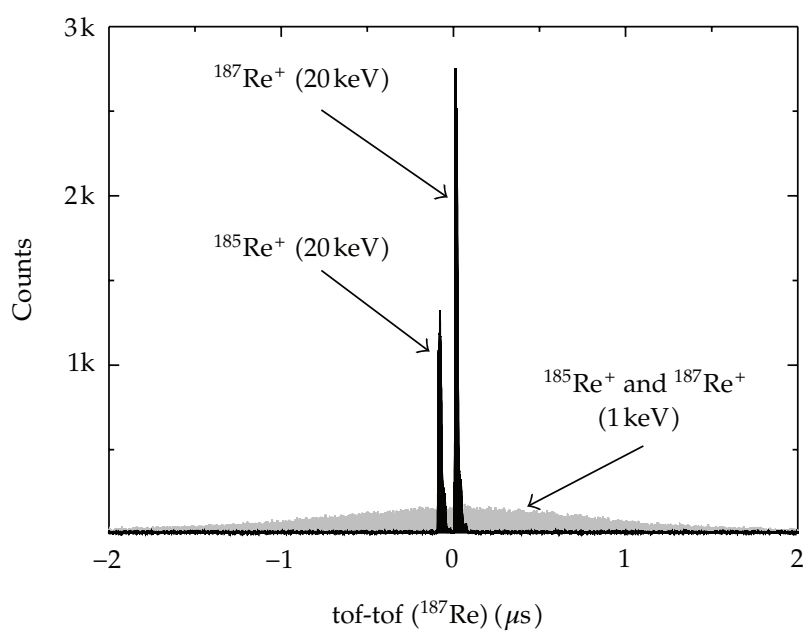

Figure 5: Time-of-flight (tof) signal of $\mathrm{Re}^{+}$ions produced with the laser desorption ion source at two different kinetic energies: $20 \mathrm{keV}$ (black) and $1 \mathrm{keV}$ (grey). Both distributions result from the accumulation of 200 cycles. In order to observe them in the same panel, the tof at $20 \mathrm{keV}$ is given with respect to the tof of the ${ }^{187} \operatorname{Re}^{+}$ions $(18 \mu \mathrm{s})$, and the one at $1 \mathrm{keV}$ with respect to the tof of the $\operatorname{Re}^{+}$ions distribution $(83 \mu \mathrm{s})$. The rhenium foil with natural abundance of $37.4 \%$ for ${ }^{185} \mathrm{Re}$ and $62.6 \%$ for ${ }^{187} \mathrm{Re}$, as can be observed from the peak distribution at $20 \mathrm{keV}$, was prepared in the target lab of GSI-Darmstadt (Germany).

measurements on very short-lived nuclei using an Advanced Trapping System) to be built at the future facility for Antiprotons and Ion Research (FAIR) in Darmstadt [36]. This Penning trap will serve to prepare the ion beam coming from the laser-desorption ion source using the buffer-gas cooling technique [31], before injection into the precision Penning trap where measurements will take place. This trap allows for isobaric separation which will be important if finally rhenium and osmium ions are extracted simultaneously from the same sample. The measurement trap is hyperbolical and identical to the precision Penning trap at TRIGATRAP [77]. This trap will allow for high-precision mass measurements using the tof technique during the first stage of the project and will be replaced in the second stage by the Quantum Sensor developed in Granada. The precision Penning trap will be operated at liquid helium temperature $(4 \mathrm{~K})$ by means of a cryocooler. The two-stage pulsed-tube cold head is located in a vacuum cross in front of the superconducting magnet to cool down the traps by thermal contact. The transfer section comprises electrostatic lenses and quadrupole deflectors and is identical to the transfer section at SHIPTRAP [53, 78]. All the beamline is housed in CF160 vacuum chambers and will be operated in high vacuum by means of three turbo molecular pumps, two of them with a pumping speed of $8001 /$ s backed by roughing pumps with pumping speeds of $35 \mathrm{~m}^{3} / \mathrm{h}$ located at both sides of the superconducting magnet. The third turbo pump, has a pumping speed of $3351 / \mathrm{s}$ and is backed by a primary pump with $3.8 \mathrm{~m}^{3} / \mathrm{h}$.

\subsection{The Laser System for ${ }^{40} \mathrm{Ca}^{+}$}

The principle of laser cooling was briefly underlined in a previous section. Since in this experiment the electronic detection will be exchanged by photon counting, it is important to show the setup to test the laser cooling of ${ }^{40} \mathrm{Ca}^{+}$in the way described previously in [27] and based on references therein. The layout of this setup is shown in Figure 7. This ion species 


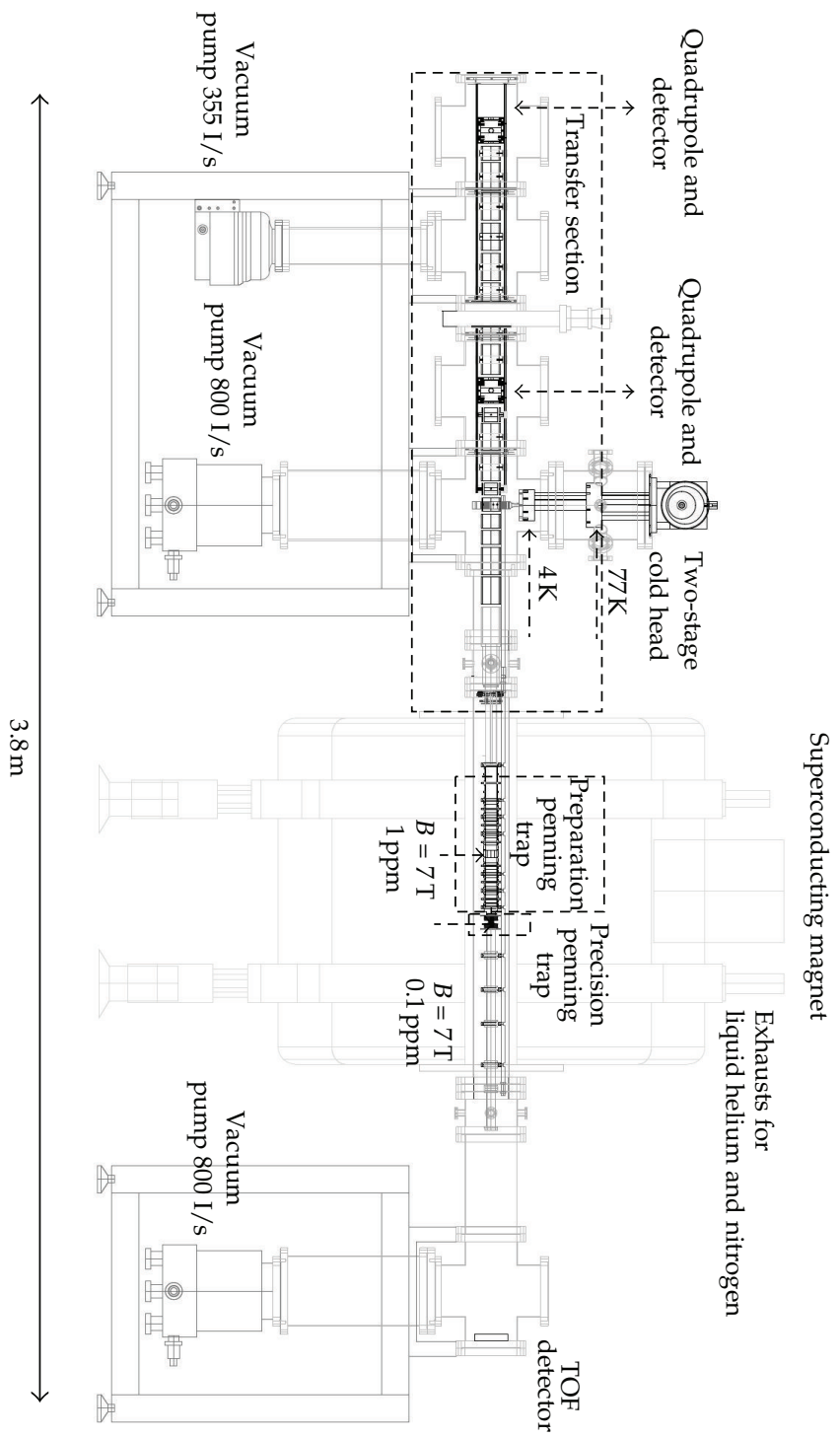

Figure 6: Layout of the Penning-trap beamline to perform high-precision mass measurements. The beamline will be coupled to an existing laser-desorption ion source.

has a transition at $\lambda=397 \mathrm{~nm}$ for Doppler cooling $\left(4^{2} S_{1 / 2} \rightarrow 4^{2} P_{1 / 2}\right)$ which can be addressed using a diode laser. Another diode laser at $\lambda=866 \mathrm{~nm}\left(4^{2} P_{1 / 2} \rightarrow 3^{2} D_{3 / 2}\right)$ is required in order to repump the ion when it decays to the dark state $3^{2} D_{3 / 2}$ with a branching ratio of $7 \%$. Thus, only a couple of lasers would be needed to cool the sensor ion. However, since the ion has to be stored finally in a $7 \mathrm{~T}$ magnetic field, the $S_{1 / 2}$ and $P_{1 / 2}$ levels are split in two and the $D_{3 / 2}$ level in four due to the Zeeman effect, requesting two lasers at $397 \mathrm{~nm}$ and four lasers at $866 \mathrm{~nm}$. The other two lasers at $854 \mathrm{~nm}$ shown in the figure will serve to repump from the state $3^{2} D_{5 / 2}$ to the state $4^{2} P_{1 / 2}$. This spontaneous decay has been observed with a branching ratio relative to the main decay of $4.2 \times 10^{-7} B^{2}$ ( $B$ given in Tesla) by a group in London [79]. 


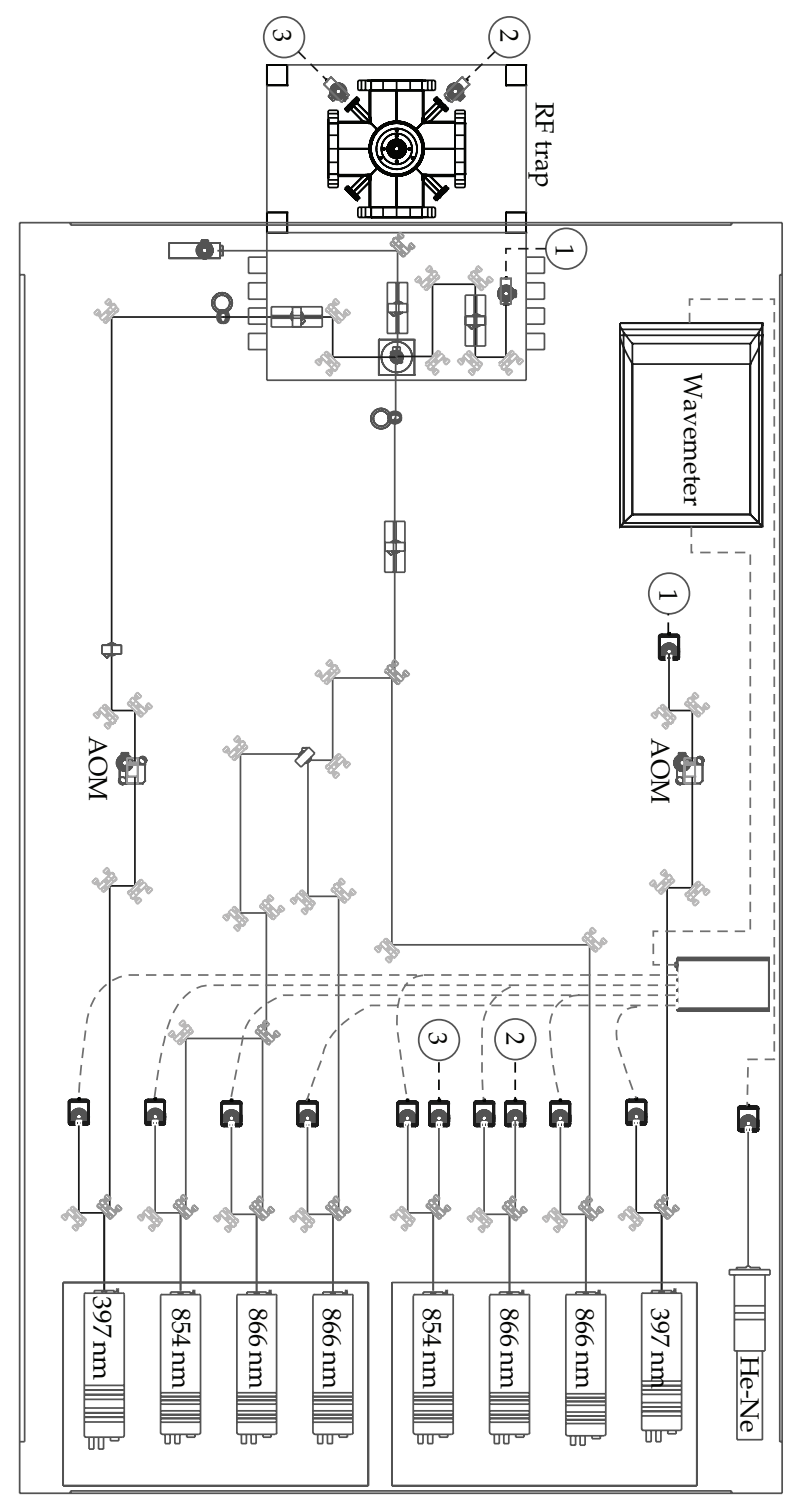

Figure 7: Optical table with the laser system and associated elements to perform laser cooling of ${ }^{40} \mathrm{Ca}^{+}$. For details see text.

As shown in Figure 7, two independent laser systems, each of them comprising four lasers, have been set up. The frequency of the lasers is locked using a very accurate wavemeter $(3 \sigma=10 \mathrm{MHz})$ and a HeNe laser at $\lambda=632 \mathrm{~nm}$ as a reference. The optics for one of the laser systems consists of mirrors and lenses to combine the different wavelengths in one line and to obtain a very strong focus $\left(\Delta r_{\mathrm{FWHM}}<10 \mu \mathrm{m}\right)$ in the center of the CF100 vaccum cross, with several view ports, where an RF trap is located. The different wavelengths for the second laser system will be transported through optical fibers directed into the chamber (see 1, 2, and 3 in Figure 7). Finally, an AOM (Acoustic Optical Modulator) will be placed in the $397 \mathrm{~nm}$ laser line to switch $\mathrm{ON}$ and $\mathrm{OFF}$ the laser in very short times to follow the detection scheme 
presented in [27]. The fluorescence will be monitored with a CCD camera located on top of the vacuum cross.

\section{Perspectives}

In this paper we have presented a new concept to improve the performance in mass spectrometry in terms of accuracy and sensitivity focusing on the application to neutrino mass spectrometry. With the setup presented, the authors envisage to yield a very accurate $Q$-value in the $\beta$-decay from ${ }^{187} \operatorname{Re}$ to ${ }^{187} \mathrm{Os}$ to contribute to the measurements to be pursued by the international MARE collaboration. The system can be also used for the determination of the $Q$-value in the $\epsilon$-decay from ${ }^{163} \mathrm{Ho}$ to ${ }^{163} \mathrm{Dy}$ provided the source is available. During the development of the system the project is structured in a way where also measurements relevant for neutrinoless double beta decay or double electron capture decay might be possible on candidates not measured yet with Penning traps. Production of any ion from solid samples can be accomplished with our universal laser desorption ion source. Ions from gas atoms can be also easily created within the preparation Penning trap.

The project officially started in November 2011, when the main equipment was ordered. The laboratory was prepared in the basement of the Faculty of Sciences and the first instruments were placed in March 2012. The beamline in Figure 6 is currently under completion. The superconducting magnet will be delivered in October 2012 and the design of the Penning-trap system has been finished and the control system of all the elements, power supplies, RF generators, and FPGA cards for pulse generation and data taking is already implemented. The coupling of the laser desorption ion source to the transfer section is currently ongoing [76]. Save for the AOM, recently ordered, the system shown in Figure 7 is completed so as to start the laser-cooling experiment. By the end of the year 2012, both setups should be in operation, so that we can start developing the Quantum Sensor within a time period of two years. For this purpose a third independent setup will be built to test the electronic coupling while mass measurements using the tof technique can be accomplished in the beamline and cooling experiments to optimize the laser system can take place in the optical table. The implementation of the device in the beamline is expected for 2015. Then, the system will be first fully devoted to the measurement of the $Q$-value of the decay ${ }^{187} \operatorname{Re}$ to ${ }^{187}$ Os. We hope to match the time schedule of the MARE collaboration.

\section{Acknowledgments}

The construction of the device described in this paper has been recently started and it is funded by the European Research Council within the ERC-2011-StG call (contract no. 268648TRAPSENSOR). Besides the applications for neutrino mass spectrometry, the device has been also conceived for applications in the field of nuclear physics. During the conception of the project, D. Rodríguez acknowledges funding from the Spanish Ministry of Science and Innovation (now integrated in the Ministry for Economy and Competitiveness) through the projects FPA2009-14091-C02-02 and FPA2010-14803. D. Rodríguez would like to thank especially H.-Jürgen Kluge for discussions on the concept of the Quantum Sensor, and Richard Thompson and Danny Segal for showing their laser labs in London and for many discussions which helped to start setting up the laser system in Granada. D. Rodríguez would like to thank also Reiner Blatt, Markus Heinrich and Michael Brownnut for showing the laser labs in Innsbruck. The authors warmly thank Rodolfo Sánchez who has set up the laser optics and the locking-frequency system with the wavemeter, Michael Block who provided 
the blueprints for many components in the beamline, and Dennis Renisch for his active participation in the measurements carried out with the MALDI in July 2011, which will be reported in a separated paper. J. M. Cornejo wants to thank the Centre for Particles, Astroparticles and Nuclear Physics in Spain for his contract. The authors would like to thank the target lab at GSI-Darmstadt for the preparation of the rhenium foil. The MALDI apparatus is a long-term loan from the University of Mainz. Klaus Wendt and Norbert Trautmann, are especially acknowledged for taking care of this in Mainz. Ignacio Porras is also especially acknowledged for providing a data acquisition module for the MALDI apparatus funded by the GREIB (Granada Research Initiative in Bio-Health) project at the University of Granada to the FQM220 group. Finally, the authors would like to thank the Vice-Rectorate for Infrastructure, the Office for International Projects, and the Department of Atomic Molecular and Nuclear Physics at the University of Granada for their contributions to the construction of the laboratory.

\section{References}

[1] Q. R. Ahmad, R. C. Allen, T. C. Andersen et al., "Direct evidence for neutrino flavor transformation from neutral-current interactions in the Sudbury Neutrino Observatory," Physical Review Letters, vol. 89, no. 1, Article ID 011301, 6 pages, 2002.

[2] M. Maltoni, T. Schwetz, M. Tórtola, and J. W. F. Valle, "Status of global fits to neutrino oscillations," New Journal of Physics, vol. 6, pp. 1-37, 2004.

[3] T. Schwetz, M. Tórtola, and J. W. F. Valle, "Three-flavour neutrino oscillation update," New Journal of Physics, vol. 10, Article ID 113011, 2008.

[4] H. Nunokawa, S. Parke, and J. W. F. Valle, “CP violation and neutrino oscillations," Progress in Particle and Nuclear Physics, vol. 60, no. 2, pp. 338-402, 2008.

[5] E. W. Otten and C. Weinheimer, "Neutrino mass limit from tritium $\beta$-decay," Reports on Progress in Physics, vol. 71, no. 8, Article ID 086201, 2008.

[6] F. Gatti, M. Ribeiro Gomes, R. Vaccarone, G. Gallinaro, L. Ferrari, and D. Bagliani, "Study of sensitivity improvement for MARE-1 in Genoa," Journal of Low Temperature Physics, vol. 151, no. 3-4, pp. 603-606, 2008.

[7] J. Bernabeu, A. De Rújula, and C. Jarlskog, "Neutrinoless double electron capture as a tool to measure the electron neutrino mass," Nuclear Physics, Section B, vol. 223, no. 1, pp. 15-28, 1983.

[8] F. Šimkovic, A. Faessler, H. Müther, V. Rodin, and M. Stauf, “0v $\beta \beta$-decay nuclear matrix elements with self-consistent short-range correlations," Physical Review C, vol. 79, no. 5, Article ID 055501, 10 pages, 2009.

[9] S. Rainville, J. K. Thompson, and D. E. Pritchard, “An ion balance for ultra-high-precision atomic mass measurements," Science, vol. 303, no. 5656, pp. 334-338, 2004.

[10] R. S. Van Dyck Jr., D. B. Pinegar, S. Van Liew, and S. L. Zafonte, "The UW-PTMS: systematic studies, measurement progress, and future improvements," International Journal of Mass Spectrometry, vol. 251, no. 2-3, pp. 231-242, 2006.

[11] A. S. Barabash, “Double $\beta$-decay: historical review of 75 years of research," Physics of Atomic Nuclei, vol. 74, no. 4, pp. 603-613, 2011.

[12] S. Rahaman, V.-V. Elomaa, T. Eronen et al., "Accurate $Q$-value for the ${ }^{112}$ Sn double- $\beta$-decay and its implication for the search of the neutrino mass," Physical Review Letters, vol. 103, no. 4, Article ID 042501, 4 pages, 2009.

[13] S. Eliseev, C. Roux, K. Blaum et al., "Resonant enhancement of neutrinoless double-electron capture in ${ }^{152}$ Gd," Physical Review Letters, vol. 106, no. 5, Article ID 052504, 4 pages, 2011.

[14] J. Angrik et al., "KATRIN design report," Wissenschaftliche Berichte FZKA 7090, 2005.

[15] F. Gatti et al., "MARE Technical Design Report," 2006, http://mare.dfm.uninsubria.it/frontend/exec .php.

[16] V. M. Lobashev, "Study of the tritium beta-spectrum in experiment 'Troitsk v-mass'," Progress in Particle and Nuclear Physics, vol. 48, no. 1, pp. 123-131, 2002.

[17] Ch. Kraus, B. Bornschein, L. Bornschein et al., "Final results from phase II of the Mainz neutrino mass searchin tritium $\beta$-decay," European Physical Journal C, vol. 40, no. 4, pp. 447-468, 2005. 
[18] Sz. Nagy, T. Fritioff, M. Björkhage, I. Bergström, and R. Schuch, “On the Q-value of the tritium $\beta$ decay," Europhysics Letters, vol. 74, no. 3, pp. 404-410, 2006.

[19] M. Sisti, C. Arnaboldi, C. Brofferio et al., "New limits from the Milano neutrino mass experiment with thermal microcalorimeters," Nuclear Instruments and Methods in Physics Research, Section A, vol. 520, no. 1-3, pp. 125-131, 2004.

[20] M. Galeazzi, F. Fontanelli, F. Gatti, and S. Vitale, "End-point energy and half-life of the ${ }^{187} \operatorname{Re} \beta$-decay," Physical Review C, vol. 63, no. 1, Article ID 014302, 7 pages, 2001.

[21] A. Monfardini, C. Arnaboldi, C. Brofferio et al., "The microcalorimeter arrays for a rhenium experiment (MARE): a next-generation calorimetric neutrino mass experiment," Nuclear Instruments and Methods in Physics Research, Section A, vol. 559, no. 2, pp. 346-348, 2006.

[22] P. C.-O. Ranitzsch, J.-P. Porst, S. Kempf et al., "Development of metallic magnetic calorimeters for high precision measurements of calorimetric ${ }^{187}$ Re and ${ }^{163}$ Ho Spectra," Journal of Low Temperature Physics, vol. 167, no. 5-6, pp. 1004-1014, 2012.

[23] I. Bergström, C. Carlberg, T. Fritioff, G. Douysset, J. Schönfelder, and R. Schuch, “SMILETRAPa Penning trap facility for precision mass measurements using highly charged ions," Nuclear Instruments and Methods in Physics Research, Section A, vol. 487, no. 3, pp. 618-651, 2002.

[24] C. Diehl, K. Blaum, M. Höcker et al., "Progress with the MPIK/UW-PTMS in Heidelberg," Hyperfine Interactions, vol. 199, pp. 291-300, 2011.

[25] http://www.physics.fsu.edu/research/atomic.html\#penning.

[26] F. Gatti, P. Meunier, C. Salvo, and S. Vitale, "Calorimetric measurement of the ${ }^{163}$ Ho spectrum by means of a cryogenic detector," Physics Letters, Section B, vol. 398, no. 3-4, pp. 415-419, 1997.

[27] D. Rodríguez, "A quantum sensor for high-performance mass spectrometry," Applied Physics B, vol. 107, no. 4, pp. 1031-1042, 2011.

[28] L. S. Brown and G. Gabrielse, "Geonium theory: physics of a single electron or ion in a Penning trap," Reviews of Modern Physics, vol. 58, no. 1, pp. 233-311, 1986.

[29] K. Blaum, "High-accuracy mass spectrometry with stored ions," Physics Reports, vol. 425, no. 1, pp. 1-78, 2006.

[30] D. J. Wineland and H. G. Dehmelt, "Principles of the stored ion calorimeter," Journal of Applied Physics, vol. 46, no. 2, pp. 919-930, 1975.

[31] G. Savard, S. Becker, G. Bollen et al., "A new cooling technique for heavy ions in a Penning trap," Physics Letters A, vol. 158, no. 5, pp. 247-252, 1991.

[32] W. M. Itano and D. J. Wineland, "Laser cooling of ions stored in harmonic and Penning traps," Physical Review A, vol. 25, no. 1, pp. 35-54, 1982.

[33] D. J. Larson, J. C. Bergquist, J. J. Bollinger, W. M. Itano, and D. J. Wineland, "Sympathetic cooling of trapped ions: a laser-cooled two-species nonneutral ion plasma," Physical Review Letters, vol. 57, no. 1, pp. 70-73, 1986.

[34] G. Gabrielse, "The true cyclotron frequency for particles and ions in a Penning trap," International Journal of Mass Spectrometry, vol. 279, no. 2-3, pp. 107-112, 2009.

[35] M. König, G. Bollen, H. J. Kluge, T. Otto, and J. Szerypo, “Quadrupole excitation of stored ion motion at the true cyclotron frequency," International Journal of Mass Spectrometry and Ion Processes, vol. 142, no. 1-2, pp. 95-116, 1995.

[36] D. Rodríguez, K. Blaum, W. Nörtershäuser et al., "MATS and LaSpec: high-precision experiments using ion traps and lasers at FAIR," European Physical Journal, vol. 183, no. 1, pp. 1-123, 2010.

[37] D. L. Farnham, R. S. Van Dyck, and P. B. Schwinberg, "Determination of the electron's atomic mass and the proton/electron mass ratio via penning trap mass spectroscopy," Physical Review Letters, vol. 75, no. 20, pp. 3598-3601, 1995.

[38] G. Gabrielse, A. Khabbaz, D. S. Hall, C. Heimann, H. Kalinowsky, and W. Jhe, "Precision mass spectroscopy of the antiproton and proton using simultaneously trapped particles," Physical Review Letters, vol. 82, no. 16, pp. 3198-3201, 1999.

[39] S. George, S. Baruah, B. Blank et al., "Ramsey method of separated oscillatory fields for high-precision penning trap mass spectrometry," Physical Review Letters, vol. 98, no. 16, Article ID 162501, 2007.

[40] R. Ringle, G. Bollen, P. Schury, S. Schwarz, and T. Sun, "Octupolar excitation of ion motion in a Penning trap-A study performed at LEBIT," International Journal of Mass Spectrometry, vol. 262, no. 1-2, pp. 33-44, 2007.

[41] S. Eliseev, M. Block, A. Chaudhuri et al., "Octupolar excitation of ions stored in a Penning trap mass spectrometer-A study performed at SHIPTRAP," International Journal of Mass Spectrometry, vol. 262, no. 1-2, pp. 45-50, 2007. 
[42] R. M. Weisskoff, G. P. Lafyatis, K. R. Boyce, E. A. Cornell, R. W. Flanagan, and D. E. Pritchard, "rf SQUID detector for single-ion trapping experiments," Journal of Applied Physics, vol. 63, no. 9, pp. 4599-4604, 1988.

[43] J. Ketelaer, K. Blaum, M. Block et al., "Recent developments in ion detection techniques for Penning trap mass spectrometry at TRIGA-TRAP," European Physical Journal A, vol. 42, no. 3, pp. 311-317, 2009.

[44] E. A. Cornell, R. M. Weisskoff, K. R. Boyce, R. W. Flanagan, G. P. Lafyatis, and D. E. Pritchard, "Singleion cyclotron resonance measurement of $M\left(\mathrm{CO}^{+}\right) / M\left(\mathrm{~N} 2^{+}\right)$," Physical Review Letters, vol. 63, no. 16, pp. 1674-1677, 1989.

[45] E. A. Cornell, R. M. Weisskoff, K. R. Boyce, and D. E. Pritchard, “Mode coupling in a Penning trap: $\pi$ pulses and a classical avoided crossing," Physical Review A, vol. 41, no. 1, pp. 312-315, 1990.

[46] E. A. Cornell, Mass spectroscopy using single ion cyclotron resonance [Ph.D. thesis], Massachusetts Institute of Technology, 1990.

[47] D. J. Heinzen and D. J. Wineland, "Quantum-limited cooling and detection of radio-frequency oscillations by laser-cooled ions," Physical Review A, vol. 42, no. 5, pp. 2977-2994, 1990.

[48] M. P. Bradley, J. V. Porto, S. Rainville, J. K. Thompson, and D. E. Pritchard, "Penning trap measurements of the masses of ${ }^{133} \mathrm{Cs},{ }^{87,85} \mathrm{Rb}$, and ${ }^{23} \mathrm{Na}$ with uncertainties $\leq 0.2 \mathrm{ppb}$," Physical Review Letters, vol. 83, no. 22, pp. 4510-4513, 1999.

[49] W. H. Furry, "On transition probabilities in double beta-disintegration," Physical Review, vol. 56, no. 12, pp. 1184-1193, 1939.

[50] R. G. Winter, "Double K capture and single K capture with positron emission," Physical Review, vol. 100, no. 1, pp. 142-144, 1955.

[51] E. Majorana, "Teoria simmetrica dell'elettrone e del positrone," Il Nuovo Cimento, vol. 14, no. 4, pp. 171-184, 1937.

[52] V. S. Kolhinen, S. Kopecky, T. Eronen et al., "JYFLTRAP: a cylindrical Penning trap for isobaric beam purification at IGISOL," Nuclear Instruments and Methods in Physics Research, Section A, vol. 528, no. 3, pp. 776-787, 2004.

[53] S. Rahaman, M. Block, D. Ackermann et al., “On-line commissioning of SHIPTRAP," International Journal of Mass Spectrometry, vol. 251, no. 2-3, pp. 146-151, 2006.

[54] M. Mukherjee, D. Beck, K. Blaum et al., "ISOLTRAP: an on-line Penning trap for mass spectrometry on short-lived nuclides," The European Physical Journal A, vol. 35, no. 1, pp. 1-29, 2008.

[55] M. Redshaw, E. Wingfield, J. McDaniel, and E. G. Myers, "Mass and double $\beta$-decay $Q$-value of ${ }^{136} \mathrm{Xe},{ }^{\prime \prime}$ Physical Review Letters, vol. 98, no. 5, Article ID 053003, 4 pages, 2007.

[56] D. Fink, J. Barea, D. Beck et al., " $Q$-value and half-lives for the double- $\beta$-decay nuclide ${ }^{110} P d$, , Physical Review Letters, vol. 108, no. 6, Article ID 062502, 4 pages, 2012.

[57] J. Suhonen and O. Civitarese, "Weak-interaction and nuclear-structure aspects of nuclear double $\beta$ decay," Physics Report, vol. 300, no. 3-4, pp. 123-214, 1998.

[58] A. S. Barabash, "Double $\beta$-decay: present status," Physics of Atomic Nuclei, vol. 73, no. 1, pp. 162-178, 2010.

[59] G. Audi, A. H. Wapstra, and C. Thibault, "The Ame2003 atomic mass evaluation-(II). Tables, graphs and references," Nuclear Physics A, vol. 729, no. 1, pp. 337-676, 2003.

[60] G. Douysset, T. Fritioff, C. Carlberg, I. Bergström, and M. Björkhage, "Determination of the ${ }^{76} \mathrm{Ge}$ double $\beta$-decay $Q$-value," Physical Review Letters, vol. 86, no. 19, pp. 4259-4262, 2001.

[61] V. S. Kolhinen, V. V. Elomaa, T. Eronen et al., "Accurate $Q$-value for the ${ }^{74}$ Se double-electron-capture decay," Physics Letters, Section B, vol. 684, no. 1, pp. 17-21, 2010.

[62] S. Eliseev, D. Nesterenko, K. Blaum et al., " $Q$-values for neutrinoless double-electron capture in ${ }^{96} \mathrm{Ru}$, ${ }^{162} \mathrm{Er}$, and ${ }^{168} \mathrm{Yb}$," Physical Review C, vol. 83, no. 3, Article ID 038501, 3 pages, 2011.

[63] S. Rahaman, V. V. Elomaa, T. Eronen et al., " $Q$-values of the ${ }^{76} \mathrm{Ge}$ and ${ }^{100}$ Mo double $\beta$-decays," Physics Letters, Section B, vol. 662, no. 2, pp. 111-116, 2008.

[64] S. Rahaman, V.-V. Elomaa, T. Eronen et al., "Double $\beta$-decay $Q$-values of ${ }^{116} \mathrm{Cd}$ and ${ }^{130} \mathrm{Te}, "$ Physics Letters, Section B, vol. 703, no. 4, pp. 412-416, 2011.

[65] M. Redshaw, B. J. Mount, E. G. Myers, and F. T. Avignone II, “Masses of ${ }^{130} \mathrm{Te}$ and ${ }^{130} \mathrm{Xe}$ and double$\beta$-decay $Q$-value of ${ }^{130}$ Te," Physical Review Letters, vol. 102, no. 21, Article ID 212502, 4 pages, 2009.

[66] V. S. Kolhinen, T. Eronen, D. Gorelov et al., "On the resonant neutrinoless double-electron-capture decay of ${ }^{136}$ Ce," Physics Letters, Section B, vol. 697, no. 2, pp. 116-120, 2011.

[67] V. S. Kolhinen et al., "Double- $\beta$-decay $Q$-value of ${ }^{150} \mathrm{Nd}$," Physical Review C, vol. 82, Article ID 022501 , 4 pages, 2010.

[68] S. Eliseev, M. Goncharov, K. Blaum et al., "Multiple-resonance phenomenon in neutrinoless doubleelectron capture," Physical Review C, vol. 84, no. 1, Article ID 012501, 4 pages, 2011. 
[69] S. Eliseev, C. Roux, K. Blaum et al., “Octupolar-excitation Penning-trap mass spectrometry for $Q$-value measurement of double-electron capture in ${ }^{164} \mathrm{Er}$," Physical Review Letters, vol. 107, no. 15, Article ID 152501, 5 pages, 2011.

[70] C. Droese, K. Blaum, M. Block et al., "Probing the nuclide ${ }^{180} \mathrm{~W}$ for neutrinoless double-electron capture exploration," Nuclear Physics A, vol. 875, pp. 1-7, 2012.

[71] G. Drexlin et al., "Absolute Neutrino Mass," This issue.

[72] A. Nucciotti et al., "The first phase of the MARE project in Milano," Nuclear Instruments and Methods A, vol. 617, pp. 509-510, 2010.

[73] G. Gabrielse, "Relaxation calculation of the electrostatic properties of compensated Penning traps with hyperbolic electrodes," Physical Review A, vol. 27, no. 5, pp. 2277-2290, 1983.

[74] K. Blaum, Y. N. Novikov, and G. Werth, "Penning traps as a versatile tool for precise experiments in fundamental physics," Contemporary Physics, vol. 51, no. 2, pp. 149-175, 2010.

[75] Yu. N. Novikov, private communication.

[76] A. Lorenzo et al., "Adapting a laser desorption ion source to perform precision experiments in Penning traps," in preparation.

[77] J. Ketelaer, J. Krämer, D. Beck et al., "TRIGA-SPEC: a setup for mass spectrometry and laser spectroscopy at the research reactor TRIGA Mainz," Nuclear Instruments and Methods in Physics Research, Section A, vol. 594, no. 2, pp. 162-177, 2008.

[78] M. Block, private communication.

[79] D. R. Crick, S. Donnellan, D. M. Segal, and R. C. Thompson, "Magnetically induced electron shelving in a trapped Ca ${ }^{+}$ion," Physical Review A, vol. 81, no. 5, Article ID 052503, 4 pages, 2010. 

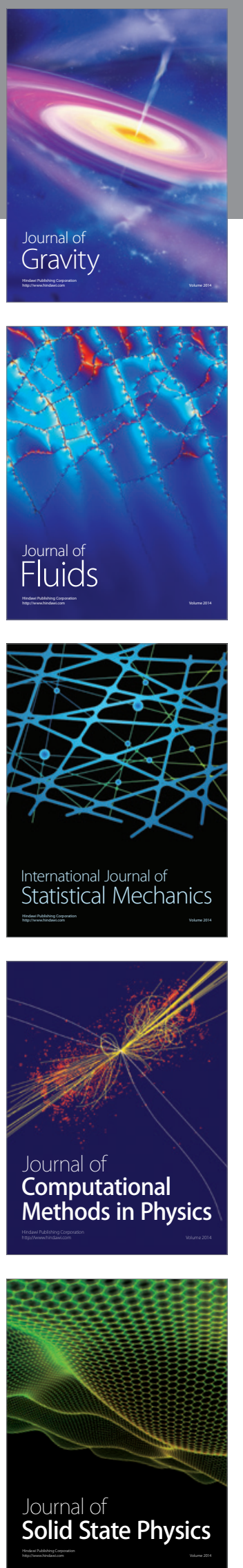

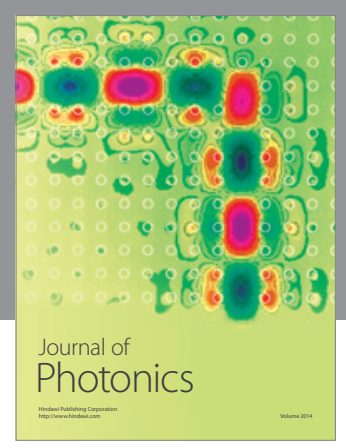

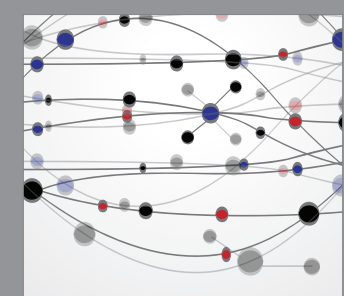

The Scientific World Journal
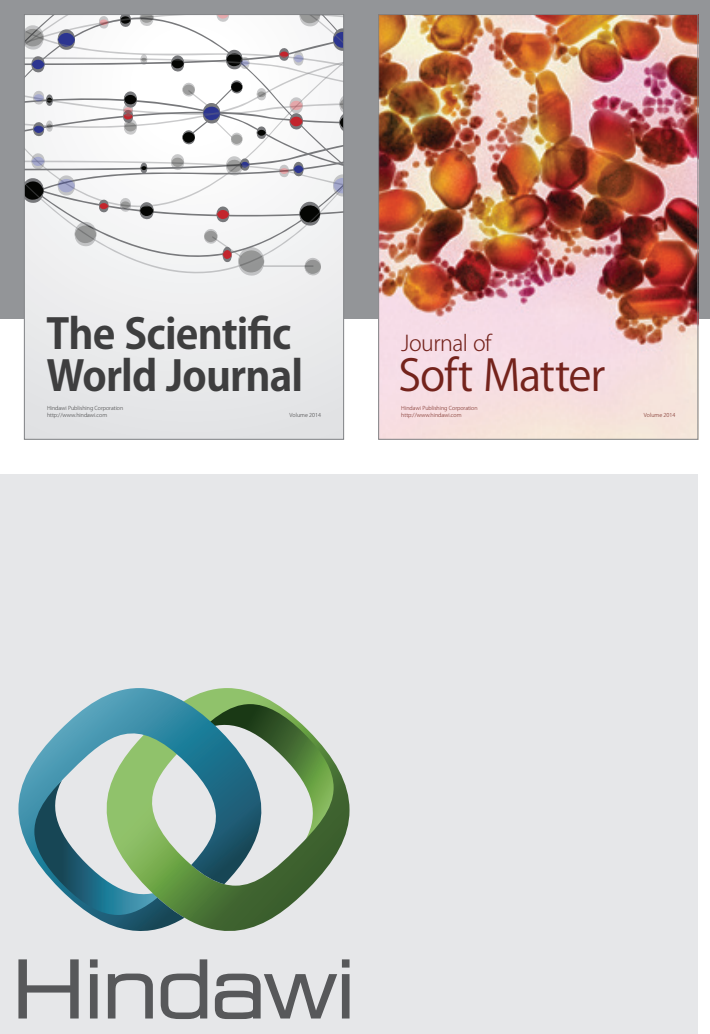

Submit your manuscripts at

http://www.hindawi.com
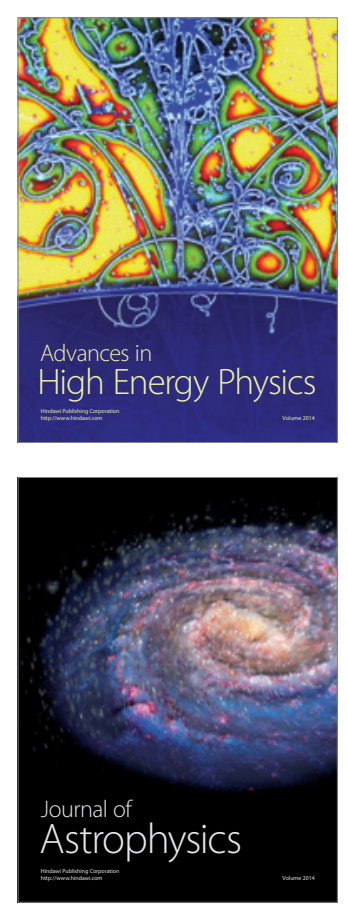
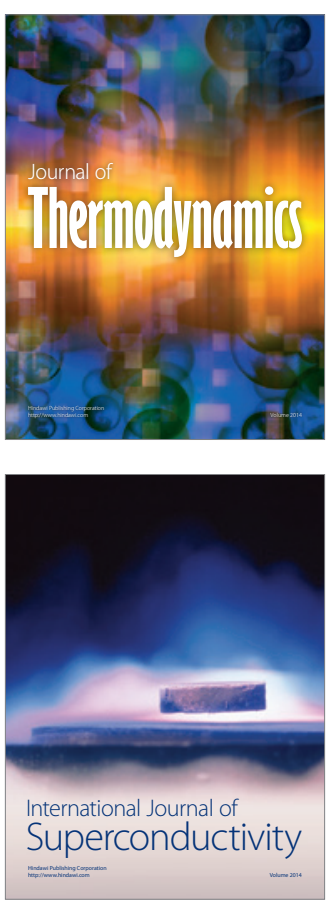
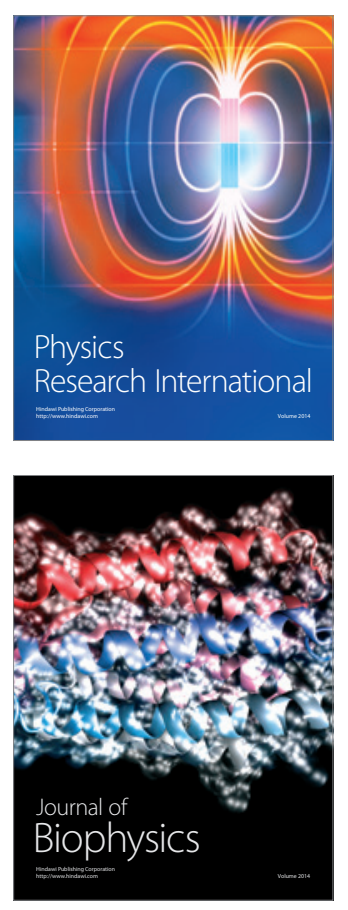
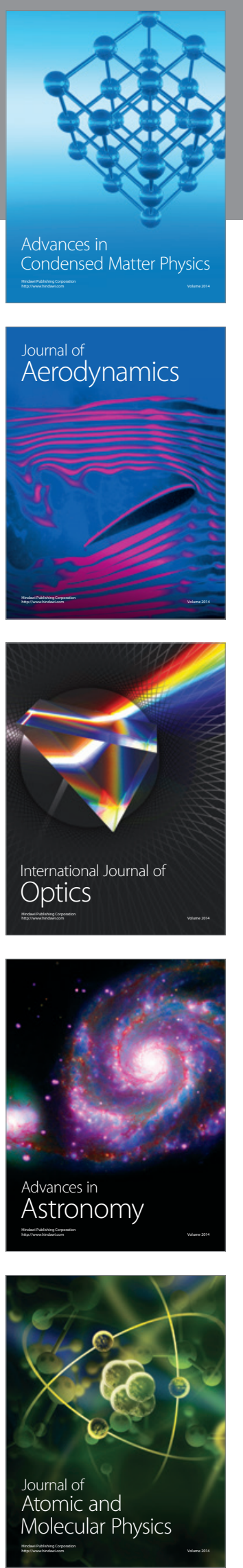